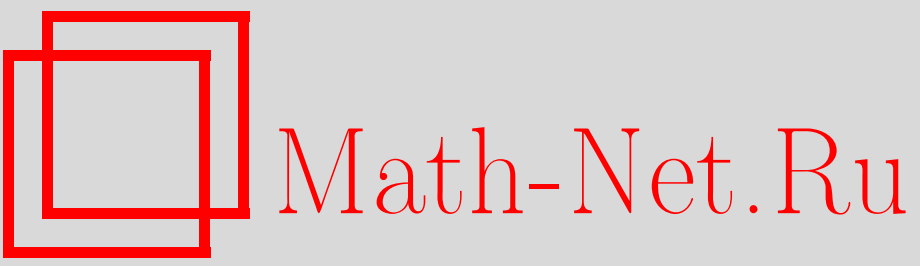

Р. Р. Булатова, Система уравнений симметричного пограничного слоя модифицированной жидкости О. А. Ладыженской, Итоги науки и техн. Сер. Соврем. мат. и ее прил. Темат. обз., 2019, том 171, 19-37

DOI: https://doi.org/10.36535/0233-6723-2019-171-19-37

Использование Общероссийского математического портала Math-Net.Ru подразумевает, что вы прочитали и согласны с пользовательским соглашением

http: //www . mathnet.ru/rus/agreement

Параметры загрузки:

IP: 35.173 .137 .237

26 апреля 2023 г., $15: 36: 44$ 


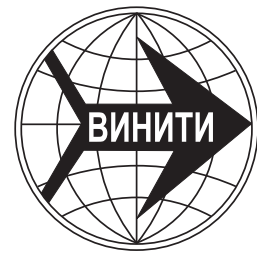

ИТОГИ НАУКИ И ТЕХНИКИ.

Современная математика и ее приложения.

Тематические обзоры.

Том 171 (2019). С. 19-37

DOI: 10.36535/0233-6723-2019-171-19-37

УДК 517.956 .45

\title{
СИСТЕМА УРАВНЕНИЙ СИММЕТРИЧНОГО ПОГРАНИЧНОГО СЛОЯ МОДИФИЦИРОВАННОЙ ЖИДКОСТИ О. А. ЛАДЫЖЕНСКОЙ
}

(c) 2019 г. $\quad$ Р. Р. БУЛАТОВА

\begin{abstract}
АннотАция. В статье изложены результаты изучения системы уравнений пограничного слоя нелинейно вязкой электропроводящей жидкости с реологическим законом, предложенным О. А. Ладыженской для несжимаемых сред. Впервые уравнения пограничного слоя для модели Ладыженской были выведены на основании аксиом Прандтля; при помощи преобразования Мизеса система уравнений пограничного слоя может быть сведена к одному квазилинейному уравнению. Основой методов, применяемых в данной работе, является преобразование Крокко, которое переводит систему уравнений пограничного слоя в квазилинейное вырождающееся параболическое уравнение, но в отличие от переменных Мизеса замена переменных Крокко позволяет изучать как стационарные, так и нестационарные уравнения.
\end{abstract}

Ключевые слова: симметричный пограничный слой, уравнения О. А. Ладыженской, преобразование Крокко, электропроводящая жидкость.

\section{O. A. LADYZHENSKAYA'S SYSTEM OF EQUATIONS OF SYMMETRIC BOUNDARY LAYER OF MODIFIED FLUID}

\author{
(c) 2019 R. R. BULATOVA
}

\begin{abstract}
In this paper, we study the system of equations of a boundary layer for a nonlinearly viscous, electrically conductive fluid described by a rheological law proposed by O. A. Ladyzhenskaya for incompressible media. The boundary-layer equations for the Ladyzhenskaya model were first obtained from Prandtl's axioms. By the Mises transform, the system of boundary-layer equations can be reduced to a single quasilinear equation. The main method used in this paper is the Crocco transform, which turns the system of boundary-layer equations into a quasilinear degenerate parabolic equation. In contrast to the Mises variables, the Crocco substitution allows one to study both stationary and nonstationary equations.
\end{abstract}

Keywords and phrases: symmetric boundary layer, O. A. Ladyzhenskaya's equations, Crocco transform, electrically conductive liquid.

AMS Subject Classification: 35K55

1. Введение. В ряде областей гидродинамики, механики и физики по сей день возникает необходимость изучения движений электропроводящих жидкостей. K таким областям относятся авиационная и судостроительная техника, разработка магнитогидродинамических генераторов электрической энергии, электромагнитных насосов, используемых для перекачки жидких металлов или плазменных ускорителей. Магнитная гидродинамика интересна нам тем, что она дополнена силами электромагнитной природы, которые, в свою очередь, позволяют управлять потоком. Например, в авиационной и судостроительной технике особенно важными являются течения при больших числах Рейнольдса, когда влияние вязкости играет значительную роль в слое, плотно 
примыкающем к поверхности обтекаемого тела. А под действием достаточно сильного магнитного поля можно предотвратить возникновение турбулентности в пограничном слое и избежать выхода его во внешний поток.

В настоящей работе рассматривается система уравнений, которая описывает динамику маловязкой электропроводящей среды в окрестности критической точки обтекаемого тела в случае двумерного симметричного потока и называется системой уравнений пограничного слоя. Критической точкой в этой теории называется точка, в которой скорость внешнего потока равна нулю. В данной статье применяется преобразование Крокко, которое приводит систему уравнений Прандтля к уравнению в конечной области с нелинейными граничными условиями. Решения этих нелинейных граничных задач построены методом прямых с дискретизацией переменной.

2. Преобразование Крокко. В случае двумерного стационарного течения модифицированная система уравнений электропроводящей жидкости имеет следующий вид:

$$
\left\{\begin{array}{l}
\nu\left(1+3 d\left(\frac{\partial u}{\partial y}\right)^{2}\right) \frac{\partial^{2} u}{\partial y^{2}}-u \frac{\partial u}{\partial x}-v \frac{\partial u}{\partial y}+B^{2}(U-u)=-U \frac{d U}{d x} \\
\frac{\partial u}{\partial x}+\frac{\partial v}{\partial y}=0 .
\end{array}\right.
$$

Здесь $\nu, d$-постоянные, зависящие от свойств жидкости, плотность жидкости $\rho$ и проводимость среды $\sigma$ предполагаются равными единице, $U(x)$ и $B(x)$ - заданные функции. Функция скорости внешнего потока $U(x)$ связана с давлением $p(x)$ и компонентой электрического поля $B(x)$ соотношением

$$
U \frac{d U}{d x}=-\frac{d p}{d x}-E \frac{B}{\sigma}-B^{2} U
$$

Система уравнений (2.1) рассматривается в области $D=\{0<x<X, 0<y<\infty\}$ с граничными условиями

$$
u(0, y)=0, \quad u(x, 0)=0, \quad v(x, 0)=v_{0}(x), \quad u(x, y) \rightarrow U(x) \quad \text { при } \quad y \rightarrow \infty,
$$

где $U(0)=0, U(x)>0$ при $x>0$. Пусть функция $U_{x}(x)$ измерима и ограничена, $U_{x}(0)>0$, функции $u_{0}(x), v_{0}(x)$ предполагаются заданными. Условия $U(0)=0$ и $u(0, y)=0$ определяют точку $x=0$ как точку, в которой происходит остановка внешнего потока жидкости и симметрический слой симметричен относительно этой точки. Далее будем предполагать, что $U(x)=x V(x)$, $V(x)>0$, и функции $V, V_{x}, v_{0}$ ограничены при $0 \leqslant x \leqslant X$.

Определение 2.1. Классическим решением задачи (2.1), (2.2) называется пара функций $u(x, y)$ и $v(x, y)$, обладающих следующими свойствами: $u$ непрерывна в замкнутой области $\bar{D}$, $v$ непрерывна в $D$ и по $y$ в $\bar{D} ; u$ и $v$ имеют в $D$ непрерывные производные, входящие в уравнение (2.1); удовлетворяют поточечно уравнениям (2.1) и граничным условиям (2.2).

Задачу (2.1), (2.2) сведем к некоторой вспомогательной краевой задаче для одного квазилинейного уравнения.

Введем новые независимые переменные $\xi, \eta$ и новую неизвестную функцию $w(\xi, \eta)$ :

$$
\xi=x, \quad \eta=\frac{u(x, y)}{U(x)}, \quad w(\xi, \eta)=\frac{u_{y}(x, y)}{U(x)} .
$$

Исключим $v$ из системы уравнений (2.1), продифференцировав эти уравнения по $y$ :

$$
\nu\left(1+3 d\left(u_{y}\right)^{2}\right) u_{y y y}+\nu u_{y y} 6 d u_{y y} u_{y}-u_{y} u_{x}-u u_{x y}-v_{y} u_{y}-v u_{y y}-B^{2} u_{y}=0 .
$$

Из системы уравнений (2.1) находим

$$
v=\frac{1}{u_{y}}\left(-u u_{x}+\nu\left(1+3 d\left(u_{y}\right)^{2}\right) u_{y y}+B^{2}(U-u)+U U_{x}\right), \quad v_{y}=-u_{x} .
$$


Из этих трех равенств выводим

$$
\begin{aligned}
\nu(1+ & \left.3 d\left(u_{y}\right)^{2}\right) u_{y y y}-\nu\left(1+3 d\left(u_{y}\right)^{2}\right) \frac{u_{y y}^{2}}{u_{y}}+\nu u_{y y}^{2} 6 d u_{y}-\left(u u_{x y}-\frac{u_{y y}}{u_{y}} u u_{x}\right)- \\
-\frac{u_{y y}}{u_{y}} U U_{x}-\frac{u_{y y}}{u_{y}} B^{2}(U-u)-B^{2} u_{y} & =\nu\left(1+3 d\left(u_{y}\right)^{2}\right) \frac{u_{y y y} u_{y}-u_{y y}^{2}}{u_{y}}-u \frac{u_{x y} u_{y}-u_{y y} u_{x}}{u_{y}}+ \\
& +6 \nu d u_{y} u_{y y}^{2}-\frac{u_{y y}}{u_{y}} U U_{x}-\frac{u_{y y}}{u_{y}} B^{2} U+\frac{u_{y y}}{u_{y}} B^{2} u-B^{2} u_{y}=0 .
\end{aligned}
$$

Вернемся к соотношениям (2.3), из которых получим

$$
\begin{gathered}
u=\eta U, \quad u_{y}=w U, \quad x=\xi, \quad u_{y y}=w_{\eta} \eta_{y} U=w_{\eta} u_{y}, \quad \frac{u_{y y}}{u_{y}}=w_{\eta}, \\
u_{y y y}=w_{\eta \eta} \eta_{y} u_{y}+w_{\eta} u_{y y}=w_{\eta \eta} \frac{u_{y}^{2}}{U}+w_{\eta} u_{y y} \\
u_{y x}=w U_{\xi}+U w_{\xi}+w_{\eta} u_{\xi}-w_{\eta} u \frac{U_{\xi}}{U}, \quad u_{y} u_{y y}^{2}=w_{\eta}^{2} w^{3} U^{3}, \\
\frac{u_{x y} u_{y}-u_{x} u_{y y}}{u_{y}}=w_{\xi} U+w U_{\xi}-w_{\eta} u_{\xi}+w_{\eta} u_{\xi}-w_{\eta} \eta U_{\xi}, \\
\frac{u_{y y y} u_{y}-u_{y y}^{2}}{u_{y}}=w_{\eta \eta} \frac{u_{y}^{2}}{U}+w_{\eta} u_{y y}-\frac{u_{y y}}{u_{y}} u_{y y}=w_{\eta \eta} \frac{u_{y}^{2}}{U}+w_{\eta} u_{y y}-w_{\eta} u_{y y}=w_{\eta \eta} \frac{u_{y}^{2}}{U^{2}} U=w_{\eta \eta} w^{2} U, \\
-\frac{u}{U}\left(\frac{u_{x y} u_{y}-u_{x} u_{y y}}{u_{y}}+\frac{u_{y y}}{u_{y}} U U_{x}\right)=-\frac{u}{U}\left(w_{\xi} U+w U_{\xi}-w_{\eta} \eta U_{\xi}\right)-w_{\eta} U_{\xi}= \\
=-\eta w_{\xi} U-\eta w U_{\xi}+\eta^{2} w_{\eta} U_{\xi}-w_{\eta} U_{\xi}=\left(\eta^{2}-1\right) w_{\eta} U_{\xi}-\eta w_{\xi} U-\eta w U_{\xi}, \\
\frac{u_{y y}}{u_{y}} B^{2} u-\frac{u_{y y}}{u_{y}} B^{2} U=w_{\eta} B^{2} u-w_{\eta} B^{2} U=w_{\eta} B^{2} \eta-w_{\eta} B^{2}=(\eta-1) B^{2} w_{\eta}, \\
B^{2} u_{y}=B^{2} \frac{u_{y}}{U} U=B^{2} w U .
\end{gathered}
$$

Преобразуем граничные условия. Имеем

$$
\begin{gathered}
v u_{y}=-u u_{x}+\nu\left(1+3 d\left(u_{y}\right)^{2}\right) u_{y y}+U U_{x}+B^{2} U-B^{2} u, \\
v \frac{u_{y}}{U}=-u \frac{u_{x}}{U}+\nu\left(1+3 d\left(u_{y}\right)^{2}\right) \frac{u_{y y}}{U}+U_{x}+B^{2}-B^{2} \frac{u}{U}, \\
v \frac{u_{y}}{U}=-u \frac{u_{x}}{U}+\nu\left(\frac{u_{y}}{U}+3 d \frac{u_{y}^{3}}{U^{3}} U^{2}\right) \frac{u_{y y}}{u_{y}}+U_{x}+B^{2}-B^{2} \frac{u}{U}, \\
v w=-u \frac{u_{\xi}}{U}+\nu\left(w+3 d U^{2} w^{3}\right) w_{\eta}+U_{\xi}+B^{2}-B^{2} \eta .
\end{gathered}
$$

Легко видеть, что $u(x, 0)=0$ и, следовательно, из $y=0$ вытекает $\eta=0$.

Таким образом, из того факта, что $y=0$, следует

$$
\nu\left(1+3 d U^{2} w^{2}\right) w w_{\eta}-v w+U_{\xi}+B^{2}=0 .
$$

В итоге, учитывая (2.2), выводим квазилинейное уравнение

$$
\nu\left(1+3 d U^{2} w^{2}\right) w^{2} w_{\eta \eta}-\eta U w_{\xi}+\left(\eta^{2}-1\right) U_{\xi} w_{\eta}-\eta U_{\xi} w+6 \nu d U^{2} w_{\eta}^{2} w^{3}+(\eta-1) B^{2} w_{\eta}-B^{2} U w=0
$$

в области $\Omega=\{0<\xi<X, 0<\eta<1\}$ с граничными условиями

$$
w(\xi, 1)=0,\left.\quad\left(\nu w w_{\eta}\left(1+3 d U^{2} w^{2}\right)-v_{0}(\xi) w+U_{\xi}+B^{2}\right)\right|_{\eta=0}=0 .
$$

Определение 2.2. Функция $w(\xi, \eta)$ называется решением задачи $(2.4),(2.5)$, если она непрерывна в $\bar{\Omega}$ и имеет непрерывные производные $w_{\xi}, w_{\eta}, w_{\eta \eta}$ в $\Omega ; w_{\eta}$ непрерывна по $\eta$ при $\eta=0$; $w$ удовлетворяет уравнению (2.4) в $\Omega$ и граничным условиям (2.5). 


\section{3. Теорема о существовании решения задачи.}

Теорема 3.1. Пусть $U$ u $v_{0}$ удовлетворяют условиям, приведенным выше. Тогда задача (2.4), (2.5) в области $\Omega$, где $X$ зависит от $U, v_{0}, \nu$, имеет решение положительное при $\eta<1$ обладающее следующими свойствами: $w(\xi, \eta)$ непрерывна $\bar{\Omega}$; ее обобщенные производные $w_{\xi}, w_{\eta}, w_{\eta \eta}$ существуют и удовлетворлют неравенствам

$$
\begin{gathered}
Y e^{-C_{1} \xi} \leqslant w \leqslant Y e^{C_{2} \xi}, \quad Y_{\eta} e^{C_{4} \xi} \leqslant w_{\eta} \leqslant Y_{\eta} e^{-C_{5} \xi}, \\
\left|w_{\xi}(\xi, \eta)\right| \leqslant C_{3} Y, \quad-C_{6} \leqslant w^{2} w_{\eta \eta} \leqslant-C_{7} .
\end{gathered}
$$

В любой замкнутой области, лежащей внутри $\Omega$, функиия $w$ и ее производные, входящие в уравнение (2.4), удовлетворяют условию Гельдера.

Доказательство существования решения задачи (2.4), (2.5) проведем на основе метода прямых, т.е. дискретизацией по $\xi$ и заменой уравнения (2.4) системой обыкновенных дифференциальных уравнений.

Для любой функции $f(\xi, \eta)$ введем обозначение

$$
f^{k}=f^{k}(\eta) \equiv f(k h, \eta), \quad h=\text { const }>0, \quad k=0,1, \ldots,[X / h] .
$$

Уравнения (2.4) с условиями (2.5) заменим системой дифференциальных уравнений

$$
\begin{aligned}
L_{k}(w):= & \nu\left(1+3 d(k h)^{2}\left(V^{k}\right)^{2}\left(w^{k}\right)^{2}\right)\left(w^{k}\right)^{2} w_{\eta \eta}^{k}-\eta k h V^{k} \frac{w^{k}-w^{k-1}}{h}+ \\
& +\left(\eta^{2}-1\right)\left(V^{k}+k h V_{\xi}^{k}\right) w_{\eta}^{k}-\eta\left(V^{k}+k h V_{\xi}^{k}\right) w^{k}+6 \nu d(k h)^{2}\left(V^{k}\right)^{2}\left(w_{\eta}^{k}\right)^{2}\left(w^{k}\right)^{3}+ \\
& \quad+(\eta-1) B^{2} w_{\eta}^{k}-B^{2} k h V^{k} w^{k}=0, \quad 0<\eta<1, \quad k=0,1, \ldots,[X / h] .
\end{aligned}
$$

с граничными условиями

$$
w^{k}(1)=0, \quad l_{k}(w)=\left.\left(\nu w^{k} w_{\eta}^{k}\left(1+3 d(k h)^{2}\left(V^{k}\right)^{2}\left(w^{k}\right)^{2}\right)-v_{0}^{k} w^{k}+\left(V^{k}+k h V_{\xi}^{k}\right)+B^{2}\right)\right|_{\eta=0}=0 .
$$

Докажем существование решения задачи (3.3), (3.4). В дальнейшем через $M_{i}$ и $K_{i}$ будем обозначать положительные постоянные, не зависящие от $h$.

Лемма 3.1. Задача (3.3), (3.4) имеет решение $w^{k}(\eta), k=0,1, \ldots,[X / h]$, которое непреръвно при $0 \leqslant \eta \leqslant 1$ и бесконечно дифферениируемо при $0 \leqslant \eta<1$, если $V_{x}>0$ при $0 \leqslant x \leqslant X u V, V_{x}$, $v_{0}$ ограничены при $0 \leqslant x \leqslant X$. Для этого решения справедлива оченка

$$
K_{1}(1-\eta) \leqslant w^{k}(\eta) \leqslant K_{2}(1-\eta) \sqrt{-\ln \mu(1-\eta)}
$$

nрu $k h \leqslant X$, где $h \leqslant h_{0}, h_{0}=$ const $>0, \mu=$ const, $0<\mu<1$.

Доказательство. Решение системы (3.3) с условиями (3.4) получим как предел при $\varepsilon \rightarrow 0$ решений системы

$$
\begin{aligned}
L_{\varepsilon, k}(w):=\nu( & \left.\left(1+3 d(k h)^{2}\left(V^{k}\right)^{2}\left(w^{k}\right)^{2}\right)\left(w^{k}\right)^{2}+\varepsilon\right) w_{\eta \eta}^{k}- \\
& -\eta k h V^{k} \frac{w^{k}-w^{k-1}}{h}+\left(\eta^{2}-1\right)\left(V^{k}+k h V_{\xi}^{k}\right) w_{\eta}^{k}-\eta\left(V^{k}+k h V_{\xi}^{k}\right) w^{k}+ \\
& \quad+6 \nu d(k h)^{2}\left(V^{k}\right)^{2}\left(w_{\eta}^{k}\right)^{2}\left(w^{k}\right)^{3}+(\eta-1) B^{2} w_{\eta}^{k}-B^{2} k h V^{k} w^{k}=0
\end{aligned}
$$

при $0<\eta<1, \varepsilon>0$ с условиями (3.4). Предположив, что задача (3.6), (3.4) имеет решение $w^{k}$, положительное при $\eta=0$, получим для этого решения априорную оценку снизу. Пусть $V_{1}^{k}=$ $K_{1}(1-\eta)$. Тогда

$$
L_{\varepsilon, k}\left(V_{1}\right)=K_{1}(1-\eta)\left(\left(V^{k}+k h V_{\xi}^{k}\right)+B^{2}-B^{2} k h V^{k}+6 \nu d(k h)^{2}\left(V^{k}\right)^{2} K_{1}^{2} K_{1}^{2}(1-\eta)^{2}\right)>0
$$

при $0 \leqslant \eta<1$. 
Положим

$$
\left.\lambda_{\varepsilon, k}(w) \equiv\left(\nu\left(1+3 d(k h)^{2}\left(V^{k}\right)^{2}\left(w^{k}\right)^{2}\right) w_{\eta}^{k}-v_{0}^{k}+\frac{V^{k}+k h V_{\xi}^{k}}{w^{k}}+\frac{B^{2}}{w^{k}}\right)\right|_{\eta=0}
$$

Тогда

$$
\lambda_{\varepsilon, k}\left(V_{1}\right)=\left.\left(\nu\left(1+3 d(k h)^{2}\left(V^{k}\right)^{2}\left(K_{1}\right)^{2}\right)\left(-K_{1}\right)-v_{0}^{k}+\frac{V^{k}+k h V_{\xi}^{k}}{K_{1}}+\frac{B^{2}}{K_{1}}\right)\right|_{\eta=0}>0,
$$

если $K_{1}$ достаточно мало.

Положим $y^{k}=V_{1}^{k}-w^{k}$ и покажем, что $y^{k} \leqslant 0$. Получим

$$
\begin{aligned}
& L_{\varepsilon, k}\left(V_{1}\right)-L_{\varepsilon, k}(w)=\left(\nu\left(1+3 d(k h)^{2}\left(V^{k}\right)^{2}\left(w^{k}\right)^{2}\right)\left(w^{k}\right)^{2}+\varepsilon\right) y_{\eta \eta}^{k}-\eta(k h) V^{k} \frac{y^{k}-y^{k-1}}{h}+ \\
&+\left(\eta^{2}-1\right)\left(V^{k}+k h V_{\xi}^{k}\right) y_{\eta}^{k}-\eta\left(V^{k}+k h V_{\xi}^{k}\right) y^{k}-B^{2} k h V^{k} y^{k}+(\eta-1) B^{2} y_{\eta}^{k}+ \\
&+6 \nu d(k h)^{2}\left(V^{k}\right)^{2}\left(\left(V_{1_{\eta}}^{k}\right)^{2}\left(\left(V_{1}^{k}\right)^{2}+V_{1}^{k} w^{k}+\left(w^{k}\right)^{2}\right) y^{k}+\left(w^{k}\right)^{3}\left(V_{1_{\eta}}^{k}+w_{\eta}^{k}\right) y_{\eta}^{k}\right)>0, \\
& \lambda_{\varepsilon, k}\left(V_{1}\right)-\lambda_{\varepsilon, k}(w)= \nu\left(1+3 d(k h)^{2}\left(V^{k}\right)^{2}\left(w^{k}(0)\right)^{2}\right) y_{\eta}^{k}(0)-\frac{B^{2}}{V_{1}^{k}(0) w^{k}(0)} y^{k}(0)+ \\
&+ 3 \nu d(k h)^{2}\left(V^{k}\right)^{2}\left(\left(V_{1}^{k}(0)\right)^{2}+\left(w^{k}(0)\right)^{2}\right) V_{1_{\eta}}^{k}(0) y^{k}(0)-\frac{V^{k}+k h V_{\xi}^{k}}{V_{1}^{k}(0) w^{k}(0)} y^{k}(0)>0 .
\end{aligned}
$$

Из этих неравенств и условия $y^{k}(1)=0$ следует, что $y^{k} \leqslant 0$. Действительно, рассмотрим $S^{k}=$ $y^{k} e^{-\alpha k h}$. Получим

$$
\begin{gathered}
\left(\nu\left(1+3 d(k h)^{2}\left(V^{k}\right)^{2}\left(w^{k}\right)^{2}\right)\left(w^{k}\right)^{2}+\varepsilon\right) S_{\eta \eta}^{k} e^{\alpha k h}-\eta k h V^{k} e^{\alpha k h}\left(\frac{S^{k}-S^{k-1}}{h} e^{-\alpha h}-S^{k} \alpha e^{\alpha h^{\prime}}\right)+ \\
+\left(\eta^{2}-1\right)\left(V^{k}+k h V_{\xi}^{k}\right) e^{\alpha k h} S_{\eta}^{k}-\eta\left(V^{k}+k h V_{\xi}^{k}\right) S^{k} e^{\alpha k h}+6 \nu d(k h)^{2}\left(V^{k}\right)^{2}\left(V_{1_{\eta}}^{k}+w_{\eta}^{k}\right)\left(w^{k}\right)^{3} S_{\eta}^{k} e^{\alpha k h}+ \\
+(\eta-1) B^{2} S_{\eta}^{k} e^{\alpha k h}-B^{2} k h V^{k} S^{k} e^{\alpha k h}>0
\end{gathered}
$$

при $\eta<1,0<h^{\prime}<h$, и

$$
\begin{gathered}
\left(\nu\left(1+3 d(k h)^{2}\left(V^{k}\right)^{2}\left(w^{k}\right)^{2}\right) S_{\eta}^{k} e^{\alpha k h}-\frac{V^{k}+k h V_{\xi}^{k}}{w^{k} V_{1}^{k}} S^{k} e^{\alpha k h}-\frac{B^{2}}{w^{k} V_{1}^{k}} S^{k} e^{\alpha k h}+\right. \\
\left.+\nu V_{1_{\eta}^{k}} 3 d(k h)^{2}\left(V^{k}\right)^{2}\left(V_{1}^{k}+w^{k}\right) S^{k} e^{\alpha k h}\right)\left.\right|_{\eta=0}>0 .
\end{gathered}
$$

Если $\alpha>0$ достаточно велико и $h$ достаточно мало, то коэффициент

$$
-B^{2} k h V^{k}-\eta\left(V^{k}+k h V_{\xi}^{k}\right)-\alpha e^{\alpha h^{\prime}} \eta k h V^{k}<0
$$

при $S^{k}$ в (3.7) неположителен.

Так как $U_{\xi}^{k}=\left(V^{k}+k h V_{\xi}^{k}\right)>0$ и $w^{k}, V_{1}^{k}$ положительны при $\eta=0$, то из $(3.7),(3.8)$ в силу принципа максимума следует, что $S^{k} \leqslant 0$. Действительно, в противном случае функция $S^{k}(\eta)$ при некотором $k$ имела имела бы положительный максимум при $0 \leqslant \eta<1$. При $0<\eta<1$ функция $S^{k}(\eta)$ не может принимать положительный максимум, так как в точке максимума мы имели бы

$$
S^{k}>0, \quad S_{\eta}^{k}=0, \quad S_{\eta \eta}^{k} \leqslant 0, \quad \frac{S^{k}-S^{k-1}}{h} \geqslant 0,
$$

что противоречит (3.7). При $\eta=0$ положительный максимум $S^{k}$ также не может достигаться, так как в противном случае приходим к противоречию между неравенствами $S_{\eta}^{k}(0) \leqslant 0$ и $(3.8)$. Значит, $S^{k} \leqslant 0$ и $y^{k}=V_{1}^{k}-w^{k} \leqslant 0$. Следовательно, $w^{k}(\eta) \geqslant K_{1}(1-\eta)$ при $k h \leqslant X$. 
Для доказательства существования решения задачи (3.6), (3.4) рассмотрим вместо условий (3.4) граничные условия вида

$$
w^{k}(1)=0,\left.\quad\left(\nu\left(1+3 d(k h)^{2}\left(V^{k}\right)^{2}\left(w^{k}\right)^{2}\right) w_{\eta}^{k}-v_{0}^{k}+\frac{V^{k}+k h V_{\xi}^{k}}{\psi\left(w^{k}\right)}+\frac{B^{2}}{\psi\left(w^{k}\right)}\right)\right|_{\eta=0}=0,
$$

где $\psi(w)$ - такая бесконечно дифференцируемая функция при $w \in(-\infty ;+\infty)$, что $\psi(w)=w$ при $w \geqslant K_{3}, \psi(w)=K_{3} / 2$ при $w \leqslant K_{3} / 4$ и $0 \leqslant \psi^{\prime}(w) \leqslant 1$ при $K_{3} / 4 \leqslant w \leqslant K_{3}$. Постоянная $K_{3}$ выбрана так, что

$$
w^{k}(0) \geqslant K_{3}, \quad \max \frac{\left|v_{0}\right|}{U_{\xi}}<\frac{2}{K_{3}}, \quad K_{3} \leqslant K_{1} .
$$

Пусть $\tilde{w}^{k}$-любое решение задачи (3.6), (3.9) при $0<\varepsilon \leqslant 1$. Покажем, что справедливо неравенство $\tilde{w}^{k} \geqslant V_{1}^{k}$. Положим $\tilde{y}^{k}=V_{1}^{k}-\tilde{w}^{k}$. Имеем

$$
\begin{aligned}
\left(\nu\left(1+3 d(k h)^{2}\left(V^{k}\right)^{2}\left(V_{1}^{k}\right)^{2}\right) V_{1_{\eta}}^{k}-v_{0}^{k}\right. & \left.+\frac{V^{k}+k h V_{\xi}^{k}}{\psi\left(V_{1}^{k}\right)}+\frac{B^{2}}{\psi\left(V_{1}^{k}\right)}\right)\left.\right|_{\eta=0}= \\
& =-\nu\left(1+3 d(k h)^{2}\left(V^{k}\right)^{2} K_{1}^{2}\right) K_{1}-v_{0}^{k}+\frac{V^{k}+k h V_{\xi}^{k}}{K_{1}}+\frac{B^{2}}{K_{1}}>0
\end{aligned}
$$

в силу выбора $K_{3}$ и

$$
\begin{gathered}
\left(\nu\left(1+3 d(k h)^{2}\left(V^{k}\right)^{2}\left(w^{k}\right)^{2}\right) \tilde{y}_{\eta}^{k}+\nu V_{1_{\eta}}^{k} 3 d(k h)^{2}\left(V^{k}\right)^{2}\left(V_{1}^{k}+w^{k}\right) \tilde{y}^{k}-\right. \\
\left.-\frac{V^{k}+k h V_{\xi}^{k}}{\psi\left(V^{k}\right) \psi\left(w^{k}\right)} \tilde{y}^{k}-\frac{B^{2} \tilde{y}^{k}}{\psi\left(V^{k}\right) \psi\left(w^{k}\right)}\right)\left.\right|_{\eta=0}>0, \\
\left(\nu\left(1+3 d(k h)^{2}\left(V^{k}\right)^{2}\left(w^{k}\right)^{2}\right)+\varepsilon\right)\left(w^{k}\right)^{2} \tilde{y}_{\eta \eta}^{k}-\eta k h V^{k} \frac{\tilde{y}^{k}-\tilde{y}^{k-1}}{h}+\left(\eta^{2}-1\right)\left(V^{k}+k h V_{\xi}^{k}\right) \tilde{y}_{\eta}^{k}- \\
-\eta\left(V^{k}+k h V_{\xi}^{k}\right) \tilde{y}^{k k}+6 \nu d(k h)^{2}\left(V^{k}\right)^{2}\left(w^{k}\right)^{3}\left(\tilde{y}_{\eta}^{k}\right)^{2}+(\eta-1) B^{2} \tilde{y}_{\eta}^{k}-B^{2} k h V^{k} \tilde{y}^{k}>0
\end{gathered}
$$

при $\eta<1$.

Далее, для $\tilde{S}^{k}=\tilde{y}^{k} e^{-\alpha k h}$, как и для $S^{k}$, получим, что $\tilde{S}^{k} \leqslant 0$ и $V_{1}^{k} \leqslant \tilde{w}^{k}$ при $0 \leqslant \eta \leqslant 1$, $k h \leqslant X$. Следовательно, $w^{k}(0) \geqslant V_{1}^{k}(0) \geqslant K_{4}$, и поэтому $\psi\left(\tilde{w}^{k}\right)=\tilde{w}^{k}$. Таким образом, решение $\tilde{w}^{k}(\eta)$ задачи (3.6), (3.9) является также решением задачи (3.6), (3.4), положительным при $\eta=0$. Если $\alpha>0$ достаточно велико и $h$ достаточно мало, то коэффициент

$$
D^{k}-\eta\left(V^{k}+k h V_{\xi}^{k}\right)-\alpha e^{\alpha h^{\prime}} \eta k h V^{k}<0
$$

при $S^{k}$ в (3.7) неположителен.

Так как $U_{\xi}=\left(V^{k}+k h V_{\xi}^{k}\right)>0$ и $w^{k}, V_{1}^{k}$ положительны при $\eta=0$, то из $(3.7),(3.8)$ в силу принципа максимума следует, что $S^{k} \leqslant 0$. Действительно, в противном случае функция $S^{k}(\eta)$ при некотором $k$ имела имела бы положительный максимум при $0 \leqslant \eta<1$. При $0<\eta<1$ функция $S^{k}(\eta)$ не может принимать положительный максимум, так как в точке максимума имели бы

$$
S^{k}>0, \quad S_{\eta}^{k}=0, \quad S_{\eta \eta}^{k} \leqslant 0, \quad \frac{S^{k}-S^{k-1}}{h} \geqslant 0,
$$

что противоречит (3.7). При $\eta=0$ положительный максимум $S^{k}$ также не может достигаться, так как в противном случае приходим к противоречию между неравенствами $S_{\eta}^{k}(0) \leqslant 0$ и $(3.8)$. Значит, $S^{k} \leqslant 0$ и $y^{k}=V_{1}^{k}-w^{k} \leqslant 0$. Следовательно, $w^{k}(\eta) \geqslant K_{1}(1-\eta)$ при $k h \leqslant X$. 
Для доказательства существования решения задачи (3.6), (3.4) рассмотрим вместо условий (3.4) граничные условия вида

$$
w^{k}(1)=0,\left.\quad\left(\nu\left(1+3 d(k h)^{2}\left(V^{k}\right)^{2}\left(w^{k}\right)^{2}\right) w_{\eta}^{k}-v_{0}^{k}+\frac{V^{k}+k h V_{\xi}^{k}}{\psi\left(w^{k}\right)}+\frac{B^{2}}{\psi\left(w^{k}\right)}\right)\right|_{\eta=0}=0,
$$

где $\psi(w)$ - такая бесконечно дифференцируемая функция при $w \in(-\infty ;+\infty)$, что $\psi(w)=w$ при $w \geqslant K_{3}, \psi(w)=K_{3} / 2$ при $w \leqslant K_{3} / 4$ и $0 \leqslant \psi^{\prime}(w) \leqslant 1$ при $K_{3} / 4 \leqslant w \leqslant K_{3}$. Постоянная $K_{3}$ выбрана так, что

$$
w^{k}(0) \geqslant K_{3}, \quad \max \frac{\left|v_{0}\right|}{U_{\xi}}<\frac{2}{K_{3}}, \quad K_{3} \leqslant K_{1} .
$$

Пусть $\tilde{w}^{k}$ - любое решение задачи (3.6), (3.9) при $0<\varepsilon \leqslant 1$. Покажем, что справедливо неравенство $\tilde{w}^{k} \geqslant V_{1}^{k}$. Положим $\tilde{y}^{k}=V_{1}^{k}-\tilde{w}^{k}$. Имеем

$$
\begin{aligned}
\left(\nu\left(1+3 d(k h)^{2}\left(V^{k}\right)^{2}\left(V_{1}^{k}\right)^{2}\right) V_{1_{\eta}}^{k}-v_{0}^{k}\right. & \left.+\frac{V^{k}+k h V_{\xi}^{k}}{\psi\left(V_{1}^{k}\right)}+\frac{B^{2}}{\psi\left(V_{1}^{k}\right)}\right)\left.\right|_{\eta=0}= \\
& =-\nu\left(1+3 d(k h)^{2}\left(V^{k}\right)^{2} K_{1}^{2}\right) K_{1}-v_{0}^{k}+\frac{V^{k}+k h V_{\xi}^{k}}{K_{1}}+\frac{B^{2}}{K_{1}}>0
\end{aligned}
$$

в силу выбора $K_{3}$ и

$$
\begin{gathered}
\left(\nu\left(1+3 d(k h)^{2}\left(V^{k}\right)^{2}\left(w^{k}\right)^{2}\right) \tilde{y}_{\eta}^{k}+\nu V_{1_{\eta}}^{k} 3 d(k h)^{2}\left(V^{k}\right)^{2}\left(V_{1}^{k}+w^{k}\right) \tilde{y}^{k}-\right. \\
\left.-\frac{V^{k}+k h V_{\xi}^{k}}{\psi\left(V^{k}\right) \psi\left(w^{k}\right)} \tilde{y}^{k}-\frac{B^{2} \tilde{y}^{k}}{\psi\left(V^{k}\right) \psi\left(w^{k}\right)}\right)\left.\right|_{\eta=0}>0 \\
\left(\nu\left(1+3 d(k h)^{2}\left(V^{k}\right)^{2}\left(w^{k}\right)^{2}\right)+\varepsilon\right)\left(w^{k}\right)^{2} \tilde{y}_{\eta \eta}^{k}-\eta k h V^{k} \frac{\tilde{y}^{k}-\tilde{y}^{k-1}}{h}+\left(\eta^{2}-1\right)\left(V^{k}+k h V_{\xi}^{k}\right) \tilde{y}_{\eta}^{k}- \\
-\eta\left(V^{k}+k h V_{\xi}^{k}\right) \tilde{y}^{k k}+6 \nu d(k h)^{2}\left(V^{k}\right)^{2}\left(w^{k}\right)^{3}\left(\tilde{y}_{\eta}^{k}\right)^{2}+(\eta-1) B^{2} \tilde{y}_{\eta}^{k}-B^{2} k h V^{k} \tilde{y}^{k}>0
\end{gathered}
$$

при $\eta<1$.

Далее, для $\tilde{S}^{k}=\tilde{y}^{k} e^{-\alpha k h}$, как и для $S^{k}$, получим, что $\tilde{S}^{k} \leqslant 0$ и $V_{1}^{k} \leqslant \tilde{w}^{k}$ при $0 \leqslant \eta \leqslant 1, k h \leqslant X$. Следовательно, $w^{k}(0) \geqslant V_{1}^{k}(0) \geqslant K_{4}$, и поэтому $\psi\left(\tilde{w}^{k}\right)=\tilde{w}^{k}$. Таким образом, решение $\tilde{w}^{k}(\eta)$ задачи (3.6), (3.9) является также решением задачи (3.6), (3.4), положительным при $\eta=0$.

Доказательство существования решения задачи (3.6), (3.9) при $\varepsilon>0$ проводится на основе теоремы Лере-Шаудера.

Оценим теперь решение задачи (3.6), (3.4) сверху равномерно относительно $\varepsilon$ и $h$. Пусть

$$
V_{2}^{k}=K_{2}(1-\eta) \sigma, \quad \sigma=\sqrt{-\ln \mu(1-\eta)}, \quad 0<\mu<1 .
$$

Тогда

$$
\begin{aligned}
L_{\varepsilon, k}\left(V_{2}\right)= & -\varepsilon K_{2}\left(-\frac{1}{2 \sigma(1-\eta)}-\frac{1}{4 \sigma^{3}(1-\eta)}\right)-(1-\eta)(1+\eta) K_{2}\left(V^{k}+k h V_{\xi}^{k}\right)\left(\frac{1}{2 \sigma}-\sigma\right)+ \\
+ & \left(\nu\left(1+3 d(k h)^{2}\left(V^{k}\right)^{2} K_{2}^{2}(1-\eta)^{2} \sigma^{2}\right) K_{2}^{2}(1-\eta)^{2} \sigma^{2}\right)\left(-\frac{K_{2}}{2 \sigma(1-\eta)}-\frac{K_{2}}{4 \sigma^{3}(1-\eta)}\right)- \\
& -\eta K_{2}(1-\eta) \sigma\left(V^{k}+k h V_{\xi}^{k}\right)+6 \nu d(k h)^{2}\left(V^{k}\right)^{2} K_{2}^{3}(1-\eta)^{3} \sigma^{3} K_{2}^{2}\left(\frac{1}{2 \sigma}-\sigma\right)^{2}- \\
& -(1-\eta) B^{2} K_{2}\left(\frac{1}{2 \sigma}-\sigma\right)-(1-\eta) B^{2} K_{2} \sigma<0
\end{aligned}
$$


при $0 \leqslant \eta<1$, если $K_{2}>0$ достаточно велико; $K_{2}$ не зависит от $\varepsilon$ и $h$. Далее,

$$
\begin{aligned}
\lambda_{k}\left(V_{2}^{k}\right)=\left(\nu\left(1+3 d(k h)^{2}\left(V^{k}\right)^{2} K_{2}^{2}(1-\eta)^{2} \sigma^{2}\right) K_{2}\left(\frac{1}{2 \sigma}-\sigma\right)-\right. & \\
& \left.-v_{0}^{k}+\frac{V^{k}+k h V_{\xi}^{k}}{K_{2}(1-\eta) \sigma}+\frac{B^{2}}{K_{2}(1-\eta) \sigma}\right)\left.\right|_{\eta=0}<0,
\end{aligned}
$$

если $K_{2}$ достаточно велико и $\sigma^{2}>1 / 2$ при $\eta=0$, т.е. $\mu<e^{-1 / 2}$. Из неравенств (3.11), (3.12) и условия $\left.\left(w^{k}-V_{2}^{k}\right)\right|_{\eta=1}=0$ согласно принципу максимума получаем, что

$$
w^{k}-V_{2}^{k} \leqslant 0 \quad \text { при } \quad 0 \leqslant \eta \leqslant 1, \quad 0 \leqslant x \leqslant X .
$$

Из уравнений (3.6), граничного условия (3.4) при $\eta=0$ и оценок снизу и сверху для $w^{k}$ следуют равномерные по $\varepsilon$ оценки $w_{\eta}^{k}, w_{\eta \eta}^{k}$ на любом отрезке $0 \leqslant \eta \leqslant 1-\delta, \delta=$ const $>0$. Дифференцируя уравнение (3.6) по $\eta$, устанавливаем, что производные любого порядка от функций $w^{k}$ ограничены на отрезке $0 \leqslant \eta \leqslant 1-\delta$ равномерно по $\varepsilon$.

Следовательно, из совокупности решений $w^{k}$ задачи (3.6), (3.4) при $0<\varepsilon \leqslant 1$ можно выделить такую последовательность $w^{k}$, что функции $w^{k}$ вместе с их производными любого порядка при $\varepsilon \rightarrow 0$ равномерно сходятся на отрезке $0 \leqslant \eta \leqslant 1-\delta$, причем предельная функция (в силу оценок $V \leqslant w^{k} \leqslant V_{1}$ ) непрерывна при $0 \leqslant \eta \leqslant 1$, обращается в нуль при $\eta=1$ и удовлетворяет (3.3), (3.4) при $\eta<1$. Очевидно, что оценки (3.5) верны для предельных функций $w^{k}$.

Рассмотрим вспомогательную граничную задачу при $k=0$ для обыкновенного дифференциального уравнения, решение которой будем использовать для последующих оценок решений задачи $(3.3),(3.4)$. Пусть $V(0)=a, v_{0}(0)=b$. Согласно сделанным ранее предположениям, $a>0$. Рассмотрим дифференциальное уравнение

$$
L(Y) \equiv \nu Y^{2} Y_{\eta \eta}+\left(\eta^{2}-1\right) a Y_{\eta}-\eta a Y+(\eta-1) B^{2} Y_{\eta}=0, \quad 0<\eta<1,
$$

с граничными условиями

$$
\left.l(Y) \equiv\left(\nu Y Y_{\eta}-b Y+B^{2}+a\right)\right|_{\eta=0}=0, \quad Y(1)=0
$$

Лемма 3.2. Задача (3.13), (3.14) имеет решение, обладающее следующими свойствами:

$$
\begin{gathered}
M_{2}(1-\eta) \sigma \leqslant Y(\eta) \leqslant M_{1}(1-\eta) \sigma \quad n p u \quad 0 \leqslant \eta \leqslant 1, \\
M_{1}(1-\eta)\left(\sigma-K_{4}\right) \leqslant Y(\eta) \quad n p u \quad 0<\eta_{0} \leqslant \eta<1, \\
-M_{4} \sigma \leqslant Y_{\eta}(\eta) \leqslant-M_{3} \sigma, \\
\left|Y Y_{\eta \eta}\right| \leqslant M_{5}, \quad Y Y_{\eta \eta} \leqslant-M_{6}, \\
\sigma=\sqrt{-\ln \mu(1-\eta)}, \quad \mu=\text { const }, \quad 0<\mu<1, \\
\nu M_{1}^{2}=2 a, \quad \sigma(0) \geqslant \frac{|b|}{a}+2, \quad \frac{K_{4}}{\sigma}<1 \quad \text { npu } \quad \eta>\eta_{0}=\text { const } \geqslant 0 .
\end{gathered}
$$

Лемма 3.2 доказывается аналогично тому, как доказана лемма [5, § 3.2.2], лишь с некоторыми техническими осложнениями. Мы опускаем доказательство этой леммы.

Лемма 3.3. Предположим, что $V(x)=a+x a_{1}(x), v_{0}(x)=b+x b_{1}(x)$ и функиии $a_{1}(x), a_{1_{x}}(x)$, $b_{1}(x)$ ограничены при $0 \leqslant x \leqslant X$. Тогда при $0 \leqslant k h \leqslant X$, где $X$ зависит от $V(x), v_{0}(x)$, для решения $w^{k}(\eta)$ задачи (3.3), (3.4) имеют место неравенства

$$
Y e^{-C_{1} k h} \leqslant w^{k}(\eta) \leqslant Y e^{C_{2} k h}
$$

где $Y(\eta)$ - решение задачи (3.13), (3.14). 
Доказательство. Для функций $W^{k}=Y e^{-C_{1} k h}$ имеем

$$
\begin{gathered}
L_{k}(W)=e^{-C_{1} k h}\left[\nu Y^{2} Y_{\eta \eta}\left(e^{-2 C_{1} k h}-1\right)+\nu Y^{2} Y_{\eta \eta}+3 \nu d(k h)^{2}\left(V^{k}\right)^{2} Y^{4} Y_{\eta \eta}\left(e^{-4 C_{1} k h}-1\right)+\right. \\
+3 \nu d(k h)^{2}\left(\left(V^{k}\right)^{2}-a^{2}\right) Y^{4} Y_{\eta \eta}+6 \nu d(k h)^{2}\left(V^{k}\right)^{2} Y^{3} Y_{\eta}^{2}\left(e^{-3 C_{1} k h}-1\right)+ \\
+6 \nu d(k h)^{2}\left(\left(V^{k}\right)^{2}-a^{2}\right) Y^{3} Y_{\eta}^{2}+\eta k h V^{k} Y C_{1} e^{C_{1} h^{\prime}}+\left(\eta^{2}-1\right) k h\left(a_{1}^{k}+V_{x}^{k}\right) Y_{\eta}- \\
\left.-\eta k h\left(a_{1}^{k}+V^{k}\right)+(\eta-1) B^{2} Y_{\eta}-B^{2} k h\left(\left(V^{k}\right)^{2}-a^{2}\right) Y\right]=0,
\end{gathered}
$$

где $0<h^{\prime}<h$. В силу предположений леммы имеем

$$
\left|\left(V^{k}\right)^{2}-a^{2}\right|<M_{8} \quad \text { при } \quad k h \leqslant X .
$$

Кроме того, при $k h \leqslant X$ и достаточно малом $X$

$$
e^{-2 C_{1} k h}-1 \leqslant-k h C_{1}, \quad e^{-3 C_{1} k h}-1 \leqslant-\frac{3}{2} k h C_{1}, \quad e^{-4 C_{1} k h}-1 \leqslant-2 k h C_{1},
$$

а в силу леммы 3.2

$$
\left|\left(\eta^{2}-1\right) k h\left(a_{1}^{k}+V_{\xi}^{k}\right) Y_{\eta}\right| \leqslant M_{9} k h Y .
$$

Поэтому, если $C_{1}$ достаточно велико, то имеем неравенство $L_{k}(W)>0$ при $0 \leqslant \eta<1$ и $0 \leqslant k h \leqslant X$.

Учитывая (3.14), находим, что

$$
\begin{aligned}
l_{k}(W)=\left[\nu\left(1+3 d(k h)^{2}\left(\left(V^{k}\right)^{2}-\left(a_{1}^{k}\right)^{2}\right) Y^{2}\right) Y Y_{\eta}\left(e^{-3 C_{1} k h}-1\right)-\right. & \\
& \left.-v_{0}^{k} Y\left(e^{-C_{1} k h}-1\right)-\left(v_{0}^{k}-b\right) Y+\left(a_{1}^{k}-k h V_{\xi}^{k}\right)+B^{2}\right]\left.\right|_{\eta=0} .
\end{aligned}
$$

По условию леммы

$$
\left|v_{0}^{k}-b\right| \leqslant M_{10} k h, \quad\left|a_{1}^{k}-k h V_{\xi}^{k}\right| \leqslant M_{11} k h \quad \text { при } \quad k h \leqslant X .
$$

Поэтому при достаточно большой постоянной $C_{1}$ и $X_{0}$, зависящем от $V, v_{0}$, имеем $l_{k}(W)>0$ при $0 \leqslant k h \leqslant X_{0}$.

При $0 \leqslant \eta<1$ и $k h \leqslant X$ получаем неравенства

$$
L_{k}(W)-L_{k}(w)>0, \quad l_{k}(W)-l_{k}(w)>0,
$$

откуда следует

$$
w^{k} \leqslant W=Y e^{-C_{1} k h} \quad \text { при } \quad 0 \leqslant \eta \leqslant 1, \quad 0 \leqslant k h \leqslant X_{0} .
$$

Аналогично получается оценка (3.19) сверху, так как если $W_{*}=Y e^{C_{2} k h}$ и $C_{2}$ достаточно велико, то $L_{k}\left(W_{*}\right)<0$ и $l_{k}\left(W_{*}\right)<0$ при $0 \leqslant \eta<1,0 \leqslant k h \leqslant X$.

Для обоснования предельного перехода в задаче (3.3), (3.4) при $h \rightarrow 0$ оценим величины

$$
r^{k}=\frac{w^{k}-w^{k-1}}{h}, \quad z^{k}=w_{\eta}^{k}, \quad\left(w^{k}\right)^{2} w_{\eta \eta}^{k}
$$

равномерно по $h$.

Лемма 3.4. Пусть выполненъ предположсения леммы 3.3; кроме того, $a_{1_{x x}}(x)$ и $b_{1_{x}}(x)$ ограничены. Тогда при достаточно малом $X u 0 \leqslant k h \leqslant X$ решения $w^{k}(\eta)$ задачи (3.3), (3.4) удовлетворяют неравенствам

$$
\begin{gathered}
\left|\frac{w^{k}-w^{k-1}}{h}\right| \leqslant C_{3} Y(\eta), \quad k=1,2, \ldots, \frac{X}{h}, \\
Y_{\eta} e^{C_{4} k h} \leqslant w_{\eta}^{k} \leqslant Y_{\eta} e^{-C_{5} k h}, \quad k=0,1, \ldots, \frac{X}{h}, \\
\left|\left(w^{k}\right)^{2} w_{\eta \eta}^{k}\right|<C_{6}, \quad\left(w^{k}\right)^{2} w_{\eta \eta}^{k}<-C_{7}, \quad k=0,1, \ldots, \frac{X}{h},
\end{gathered}
$$

где $Y(\eta)$ - решение задачи (3.13), (3.14). 
Доказательство. Неравенства (3.20)-(3.22) докажем по индукции. В лемме 3.2 мы доказали неравенства (3.21), (3.22) при $k=0$. Покажем, что постоянные $C_{j}$ можно подобрать независимо от $h$ так, что из выполнения неравенств (3.20)-(3.22) при некотором $k-1$ следует их справедливость при $k$, если $0 \leqslant k h \leqslant X$, где $X$ зависит от $V, v_{0}, \nu$ и не зависит от $h$.

Для удобства в уравнениях

$$
\begin{aligned}
L_{k}(w) \equiv \nu(1+ & \left.3 d(k h)^{2}\left(V^{k}\right)^{2}\left(w^{k}\right)^{2}\right)\left(w^{k}\right)^{2} w_{\eta \eta}^{k}-\eta k h V^{k} \frac{w^{k}-w^{k-1}}{h}+\left(\eta^{2}-1\right)\left(V^{k}+k h V_{\xi}^{k}\right) w_{\eta}^{k}- \\
- & \eta\left(V^{k}+k h V_{\xi}^{k}\right) w^{k}+6 \nu d(k h)^{2}\left(V^{k}\right)^{2}\left(w_{\eta}^{k}\right)^{2}\left(w^{k}\right)^{3}+(\eta-1) B^{2} w_{\eta}^{k}-B^{2} k h V^{k} w^{k}=0
\end{aligned}
$$

и

$$
\lambda_{k}(w)=\left.\left(\nu\left(1+3 d(k h)^{2}\left(V^{k}\right)^{2}\left(w^{k}\right)^{2}\right) w_{\eta}^{k}-v_{0}^{k}+\frac{V^{k}+k h V_{\xi}^{k}}{w^{k}}+\frac{B^{2}}{w^{k}}\right)\right|_{\eta=0}=0
$$

введем обозначения:

$$
\begin{gathered}
E^{k} \equiv\left(\eta^{2}-1\right)\left(V^{k}+k h V_{\xi}^{k}\right), \quad F^{k} \equiv-\eta\left(V^{k}+k h V_{\xi}^{k}\right), \\
G^{k} \equiv 3 \nu d(k h)^{2}\left(V^{k}\right)^{2}, \quad H^{k} \equiv 6 \nu d(k h)^{2}\left(V^{k}\right)^{2}, \quad I^{k} \equiv V^{k}+k h V_{\xi}^{k} .
\end{gathered}
$$

Составим уравнения, которым удовлетворяют $r^{k}$ и $z^{k}$. Для начала возьмем

$$
w_{\eta \eta}^{k}=\frac{\eta k h V^{k} r^{k}-E^{k} z^{k}-F^{k} w^{k}-H^{k}\left(z^{k}\right)^{2}\left(w^{k}\right)^{3}-(\eta-1) B^{2} z^{k}+B^{2} k h V^{k} w^{k}}{\left(\nu+G^{k}\left(w^{k}\right)^{2}\right)\left(w^{k}\right)^{2}} .
$$

Из уравнения (3.3) для $w^{k}$ вычтем уравнение (3.3) для $w^{k-1}$ и разделим полученное равенство на $h$. Имеем

$$
\begin{aligned}
& R_{k}(r) \equiv \nu\left(w^{k}\right)^{2} r_{\eta \eta}^{k}+G^{k}\left(w^{k}\right)^{4} r_{\eta \eta}^{k}+E^{k} r_{\eta}^{k}+F^{k} r^{k}-\eta \frac{k h V^{k}-(k-1) h V^{k-1}}{h} r^{k}+ \\
& +\frac{\left(w^{k}+w^{k-1}\right)\left(\left(w^{k}\right)^{2}+\left(w^{k-1}\right)^{2}\right)}{\left(\nu+G^{k-1}\left(w^{k-1}\right)^{2}\right)\left(w^{k-1}\right)^{2}}\left(\nu+G^{k-1}\right) \times \\
& \quad \times\left[\eta(k-1) h V^{k-1} r^{k-1}-E^{k-1} z^{k-1}-F^{k-1} w^{k-1}-H^{k-1}\left(z^{k-1}\right)^{2}\left(w^{k-1}\right)^{3}\right] r^{k}- \\
& -\eta(k-1) h V^{k-1} \frac{r^{k}-r^{k-1}}{h}+H^{k-1}\left(w^{k-1}\right)^{3} r^{k}\left(z^{k}+z^{k-1}\right)+\frac{H^{k}-H^{k-1}}{h}\left(z^{k}\right)^{2}\left(w^{k}\right)^{3}+ \\
& \quad+H^{k-1}\left(z^{k}\right)^{2} r^{k}\left(\left(w^{k}\right)^{2}+w^{k} w^{k-1}+\left(w^{k-1}\right)^{2}\right)+B^{2} \frac{k h V^{k}-(k-1) h V^{k-1}}{h} w^{k}= \\
& =-\frac{3 \nu d\left((k h)^{2}\left(V^{k}\right)^{2}-((k-1) h)^{2}\left(V^{k-1}\right)^{2}\right)\left(w^{k}\right)^{4}}{h\left(\nu+G^{k-1}\left(w^{k-1}\right)^{2}\right)\left(w^{k-1}\right)^{2}} \times \\
& \times\left[\eta(k-1) h V^{k-1} r^{k-1}-E^{k-1} z^{k-1}-F^{k-1} w^{k-1}-H^{k-1}\left(z^{k-1}\right)^{2}\left(w^{k-1}\right)^{3}\right]- \\
& \quad-z^{k-1} \frac{E^{k}-E^{k-1}}{h}-w^{k-1} \frac{F^{k}-F^{k-1}}{h}-(\eta-1) B^{2} r_{\eta}^{k}+B^{2} k h V^{k} r^{k-1}, \quad k \geqslant 1 .
\end{aligned}
$$

Аналогично из (3.4) получаем

$$
\begin{aligned}
\rho^{k}(r) \equiv\left(\nu r_{\eta}^{k}+G^{k-1}\left(w^{k-1}\right)^{2} r_{\eta}^{k}+G^{k-1} z^{k} r^{k}\left(w^{k}+w^{k-1}\right)+\frac{G^{k}-G^{k-1}}{h}\left(w^{k}\right)^{2} z^{k}-\right. \\
\left.-\frac{\left(I^{k}+B^{2}\right) r^{k}}{w^{k} w^{k-1}}\right)\left.\right|_{\eta=0}=\frac{v_{0}^{k}-v_{0}^{k-1}}{h}-\frac{I^{k}-I^{k-1}}{h w^{k-1}(0)}, \quad r^{k}(1)=0, \quad k \geqslant 1 .
\end{aligned}
$$


Дифференцируя уравнение (3.3) по $\eta$, для $z^{k}$ получим уравнения

$$
\begin{aligned}
P_{k}(z) \equiv & \nu\left(w^{k}\right)^{2} z_{\eta \eta}^{k}+2 \nu w^{k} z^{k} z_{\eta}^{k}+4 G^{k}\left(w^{k}\right)^{3} z^{k} z_{\eta}^{k}+G^{k}\left(w^{k}\right)^{4} z_{\eta \eta}^{k}- \\
-\eta k h V^{k} \frac{z^{k}-z^{k-1}}{h}-k h V^{k} r^{k} & +E^{k} z_{\eta}^{k}+E_{\eta}^{k} z^{k}+F^{k} z^{k}+F_{\eta}^{k} w^{k}+3 H^{k}\left(w^{k}\right)^{2}\left(z^{k}\right)^{3}+ \\
& +2 H^{k}\left(w^{k}\right)^{3} z^{k} z_{\eta}^{k}+(\eta-1) B^{2} z_{\eta}^{k}+B^{2} z^{k}+B^{2} k h V^{k} z^{k}=0 .
\end{aligned}
$$

Из граничного условия (3.4) при $\eta=0$ следует условие

$$
z^{k}(0)=\frac{v_{0}^{k}}{\left(\nu+G^{k}\left(w^{k}(0)\right)^{2}\right)}-\frac{I^{k}}{\left(\nu+G^{k}\left(w^{k}(0)\right)^{2}\right) w^{k}(0)} .
$$

Рассмотрим функцию $\varphi^{k}=C_{3} Y$ и оценим $R^{k}(\varphi)$ при условии, что неравенства $(3.20),(3.21)$ выполнены при $k-1$. Сначала заметим, что в силу результатов, полученных в лемме 3.2 , выполнено неравенство

$$
\left|\nu C_{3} Y^{2} Y_{\eta \eta}+C_{3} E^{k} Y_{\eta}+C_{3} F^{k} Y\right| \leqslant C_{3} M_{12} k h Y
$$

при $0 \leqslant k h \leqslant X$. Так как $U_{x}>0$ и $a_{1_{x x}}(0)$ ограничена по предположению леммы, то

$$
\frac{k h V^{k}-(k-1) h V^{k-1}}{h}>0
$$

при $h \leqslant h_{0}$ и достаточно малом $h_{0} ;$ кроме того, отношения

$$
\frac{(k h)^{2}\left(V^{k}\right)^{2}-((k-1) h)^{2}\left(V^{k-1}\right)^{2}}{h}, \quad \frac{E^{k}-E^{k-1}}{h}, \quad \frac{F^{k}-F^{k-1}}{h}, \quad \frac{G^{k}-G^{k-1}}{h}, \quad \frac{H^{k}-H^{k-1}}{h}
$$

ограничены.

Далее, учитывая предположения индукции, находим, что

$$
\begin{gathered}
\eta(k-1) h V^{k-1} r^{k-1}-E^{k-1} z^{k-1}-F^{k-1} w^{k-1}-H^{k-1}\left(z^{k-1}\right)^{2}\left(w^{k-1}\right)^{3}-(\eta-1) B^{2} z^{k-1} \leqslant \\
\leqslant \eta(k-1) h V^{k-1} C_{3} Y-\left(E^{k-1}-a\left(\eta^{2}-1\right)\right) z^{k-1}-\left(\eta^{2}-1\right) a Y_{\eta} e^{-C_{3} k h}-\left(F^{k-1}+\eta a\right) w^{k-1}- \\
-\eta a Y e^{C_{2} k h}-\left(B^{2}-a(\eta-1)\right) z^{k-1}-\left(H^{k-1}-6 \nu d a^{2}\right)\left(z^{k-1}\right)^{2}\left(w^{k-1}\right)^{3}- \\
-6 \nu d a^{2} Y^{3} Y_{\eta}^{2} e^{3 C_{2} k h} e^{-2 C_{5} k h} \leqslant \nu Y Y_{\eta \eta}+G^{k-1} Y^{4} Y_{\eta \eta}+M_{13} k h Y \leqslant-M_{14} Y(\eta)
\end{gathered}
$$

если $k h \leqslant X$ и $X$ достаточно мало и не зависит от $h$.

Учитывая эти замечания, получаем, что

$$
\begin{aligned}
R_{k}(\varphi)+\mid- & \frac{3 \nu d\left((k h)^{2}\left(V^{k}\right)^{2}-((k-1) h)^{2}\left(V^{k-1}\right)^{2}\right)\left(w^{k}\right)^{4}}{h\left(\nu+G^{k-1}\left(w^{k-1}\right)^{2}\right)\left(w^{k-1}\right)^{2}} \times \\
& \times\left[\eta(k-1) h V^{k-1} r^{k-1}-E^{k-1} z^{k-1}-F^{k-1} w^{k-1}-H^{k-1}\left(z^{k-1}\right)^{2}\left(w^{k-1}\right)^{3}\right]- \\
& \quad-z^{k-1} \frac{E^{k}-E^{k-1}}{h}-w^{k-1} \frac{F^{k}-F^{k-1}}{h}-B^{2}(\eta-1) r_{\eta}^{k}+B^{2} k h V^{k} r^{k-1} \mid<0
\end{aligned}
$$

при $0 \leqslant \eta<1,0 \leqslant k h \leqslant X\left(C_{3}, C_{4}, C_{5}\right)$, где $C_{3}$ достаточно велико и не зависит от $h$; здесь мы использовали неравенства

$$
\begin{gathered}
-\eta \frac{k h V^{k}-(k-1) h V^{k-1}}{h} C_{3} Y+\left|w^{k-1} \frac{F^{k}-F^{k-1}}{h}\right|<0, \\
\left(w^{k}+w^{k-1}\right)\left(\left(w^{k}\right)^{2}+\left(w^{k-1}\right)^{2}\right) C_{3} Y>\left|\frac{(k h)^{2}\left(V^{k}\right)^{2}-((k-1) h)^{2}\left(V^{k-1}\right)^{2}}{h}\right|
\end{gathered}
$$




$$
\begin{aligned}
M_{12} k h & \leqslant \frac{1}{4} \mid \frac{\left(w^{k}+w^{k-1}\right)\left(\left(w^{k}\right)^{2}+\left(w^{k-1}\right)^{2}\right)}{\left(\nu+G^{k-1}\left(w^{k-1}\right)^{2}\right)\left(w^{k-1}\right)^{2}}\left(\nu+G^{k-1}\right) \times \\
& \times\left[\eta(k-1) h V^{k-1} r^{k-1}-E^{k-1} z^{k-1}-F^{k-1} w^{k-1}-H^{k} k-1\left(z^{k-1}\right)^{2}\left(w^{k-1}\right)^{3}-(\eta-1) B^{2}\right] \mid .
\end{aligned}
$$

Постоянная $C_{3}$ не зависит от $C_{4}$ и $C_{5}$, так как если

TO

$$
M_{14} \geqslant \frac{M_{14}}{2}+\frac{M_{15} E^{k-1} z^{k-1}}{Y}
$$

$$
-\frac{C_{3} M_{15}\left(w^{k}+w^{k-1}\right)\left(\left(w^{k}\right)^{2}+\left(w^{k-1}\right)^{2}\right)\left(\nu+G^{k-1}\right) E^{k-1} z^{k-1}}{\left(\nu+G^{k-1}\left(w^{k-1}\right)^{2}\right)\left(w^{k-1}\right)^{2}}+\left|z^{k-1} \frac{E^{k}-E^{k-1}}{h}\right|<0
$$

при подходящем выборе $C_{3}$ и достаточно малом $X$.

Вычислим $\rho_{k}(\varphi)$. Имеем:

$$
\begin{aligned}
\rho^{k}(\varphi)=C_{3}\left(\nu Y_{\eta}+G^{k-1}\left(w^{k-1}\right)^{2} Y_{\eta}+G^{k-1} z^{k}\right. & Y\left(w^{k}+w^{k-1}\right)+ \\
& \left.+\frac{G^{k}-G^{k-1}}{h}\left(w^{k}\right)^{2} z^{k}-\frac{\left(I^{k}+B^{2}\right) Y}{w^{k} w^{k-1}}\right)\left.\right|_{\eta=0} \leqslant-C_{3} M_{16}
\end{aligned}
$$

при $M_{16}>0, k h \geqslant 0, k h \leqslant X$ и достаточно малом $X$. Поэтому

$$
\rho_{k}(\varphi)+\left|\frac{v_{0}^{k}-v_{0}^{k-1}}{h}-\frac{I^{k}-I^{k-1}}{h w^{k-1}(0)}\right|<0,
$$

если $C_{3}$ достаточно велико, $k h \leqslant X$ и $h \leqslant h_{0}$; здесь воспользовались тем, что по условия при малых $h$ ограничены отношения

$$
\frac{v_{0}^{k}-v_{0}^{k-1}}{h}, \quad \frac{I^{k}-I^{k-1}}{h} .
$$

Рассмотрим функции $S_{ \pm}^{k}=\varphi^{k} \pm r^{k}$ при $0 \leqslant k h \leqslant X$. Из оценок (3.23), (3.24) получаем неравенства

$$
R_{k}\left(S_{ \pm}^{k}\right)<0, \quad \rho_{k}\left(S_{ \pm}^{k}\right)<0 .
$$

Так как $S_{ \pm}^{k}(1)=0$, то из этих неравенств на основании принципа максимума находим, что $S_{ \pm}^{k}(\eta) \geqslant 0$ при $0 \leqslant \eta \leqslant 1$ и, следовательно, $\left|r^{k}\right| \leqslant C_{3} Y$.

Для оценки $Z^{k}=w_{\eta}^{k}$ рассмотрим функцию $J_{1}=Y_{\eta} e^{-C_{5} k h}$. Имеем

$$
\begin{aligned}
P_{k}\left(J_{1}\right) & =e^{-C_{5} k h}\left\{\left(\nu Y^{2} Y_{\eta \eta \eta}+2 \nu Y Y_{\eta} Y_{\eta \eta}+\left(\eta^{2}-1\right) a Y_{\eta \eta}+\eta a Y_{\eta}-a Y\right)+B^{2} Y_{\eta}+\right. \\
+ & (\eta-1) B^{2} Y_{\eta \eta}+\nu\left(\left(w^{k}\right)^{2}-Y^{2}\right) Y_{\eta \eta}+G^{k}\left(\left(w^{k}\right)^{4}-Y^{4}\right) Y_{\eta \eta \eta}+\left(E^{k}-\left(\eta^{2}-1\right) a\right) Y_{\eta \eta}+ \\
+ & \left.\left(E_{\eta}^{k}-2 \eta a\right) Y_{\eta}+\left(F^{k}-\eta a\right) Y_{\eta}+\left(B^{2}-(\eta-1) a\right) Y_{\eta \eta}+2 H^{k}\left(\left(w^{k}\right)^{3}-Y^{3}\right) Y_{\eta} Y_{\eta \eta}\right\}+ \\
- & k h V^{k} r^{k}+2 \nu Y_{\eta} Y_{\eta \eta}\left(w^{k} e^{-C_{5} k h}-Y\right) e^{-C_{5} k h}+4 G^{k} Y_{\eta} Y_{\eta \eta}\left(\left(w^{k}\right)^{3} e^{-C_{5} k h}-Y^{3}\right) e^{-C_{5} k h}+ \\
& +3 H^{k}\left(Y_{\eta}\right)^{3}\left(\left(w^{k}\right)^{2} e^{-C_{5} k h}-Y^{2}\right) e^{-C_{5} k h}+\left(B^{2}-2 \eta a\right) Y_{\eta}+\left(F_{\eta}^{k} w^{k}+a Y e^{-C_{5} k h}\right)- \\
& -B^{2} k h V^{k} Y_{\eta}+\eta k h C_{5} V^{k} e^{C_{5} h^{\prime}} e^{-C_{5} k h} Y_{\eta} .
\end{aligned}
$$

Дифференцируя уравнение (3.13) по $\eta$, получим

$$
\nu Y^{2} Y_{\eta \eta \eta}+2 \nu Y Y_{\eta} Y_{\eta \eta}+\left(\eta^{2}-1\right) a Y_{\eta \eta}+\eta a Y_{\eta}-a Y+B^{2} Y_{\eta}+(\eta-1) B^{2} Y_{\eta \eta}=0 .
$$

Из (3.26) следует, что $\left|Y^{2} Y_{\eta \eta \eta}\right| \leqslant M_{17}\left|Y_{\eta}\right|$ и что в (3.25) первое слагаемое правой части равно нулю. Слагаемые $k h V^{k} r^{k}, F_{\eta}^{k} w^{k}+a Y e^{-C_{5} k h}$ имеют порядок $k h Y$ и $C_{5} k h Y$ соответственно; остальные слагаемые в (3.25) имеют порядок $k h Y_{\eta}$. Если выбрать $C_{5}$ достаточно большим, то последние два члена в (3.25) превосходят по модулю остальные, если $k h \leqslant X$ и $X$ достаточно мало. Поэтому $P_{k}\left(J_{1}\right)<0$ при $0 \leqslant \eta \leqslant 1$ и $0 \leqslant k h \leqslant X$. 
Из неравенств (3.19) следует, что существуют такие две последовательности $\eta_{m} \rightarrow 1$ и $\bar{\eta}_{m} \rightarrow 1$ при $m \rightarrow \infty$, что

$$
\left.z^{k}\right|_{\eta=\eta_{m}} \leqslant Y_{\eta}\left(\eta_{m}\right) e^{-C_{1} k h},\left.\quad z^{k}\right|_{\eta=\bar{\eta}_{m}} \geqslant Y_{\eta}\left(\bar{\eta}_{m}\right) e^{C_{2} k h} .
$$

Пусть $C_{5}>C_{1}$. Рассмотрим разность $S^{k}=J_{1}-Z^{k}$. Из (3.27) следует, что $S^{k}\left(\eta_{m}\right) \geqslant 0$. При $k \geqslant 1, k h \leqslant X$, достаточно малом $X$ и достаточно большом $C_{5}$ имеем

$$
\begin{aligned}
& S^{k}(0)=-\frac{v_{0}^{k}-b}{\left(\nu+G^{k}\left(w^{k}(0)\right)^{2}\right)}+\frac{\left(I^{k}+B^{2}\right)}{\left(\nu+G^{k}\left(w^{k}(0)\right)^{2}\right) w^{k}(0)}- \\
& -\frac{a}{\left(\nu+G^{k}(Y(0))^{2}\right) Y(0)}+Y_{\eta}(0)\left(e^{-C^{5} k h}-1\right)>0 .
\end{aligned}
$$

Из уравнения (3.3) при $k=1$ получаем для $z^{1}=w_{\eta}^{1}$ уравнение

$$
P_{1}^{0}\left(z_{1}\right) \equiv \nu\left(w^{1}\right)^{2} z_{\eta}^{1}+G^{1}\left(w^{1}\right)^{4} z_{\eta}^{1}+E^{1} z^{1}+H^{1}\left(w^{1}\right)^{3} z^{1}+(\eta-1) B^{2} z^{1}=-J^{1} w^{1}+\eta V^{1} w^{1}+B^{2} V^{2} w^{1} .
$$

Полагая $J_{2}=Y_{\eta} e^{-C_{8} h}$, находим

$$
\begin{aligned}
P_{1}^{0}\left(z^{1}-J_{2}\right)= & e^{-C_{8} h}\left\{\nu\left(Y^{2}-\left(w^{1}\right)^{2}\right) Y_{\eta \eta}+G^{k}\left(Y^{4}-\left(w^{1}\right)^{4}\right) Y_{\eta \eta}+\right. \\
& \left.+\left(\left(\eta^{2}-1\right) a-E^{1}\right) Y_{\eta}+\left((\eta-1) a-B^{2}\right) Y_{\eta}+H^{k}\left(Y^{3}-\left(w^{1}\right)^{3}\right) Y_{\eta}\right\}- \\
& \quad-\left(F^{1} w^{1}+\eta a Y e^{-C_{8} h}\right)+\eta V^{1}\left(w^{1}-Y\right)+B^{2} V^{1}\left(w^{1}-Y\right)>0, \quad \eta_{0} \leqslant \eta<1,
\end{aligned}
$$

при достаточно большом $C_{8}$ и достаточно малом $h$. Кроме того, если выберем $C_{8} \geqslant C_{1}$, то из (3.27) получим неравенство

$$
\left.\left(z^{1}-J_{2}\right)\right|_{\eta=\eta_{m}^{1}} \leqslant 0
$$

Как и при доказательстве леммы 3.2 , находим, что $z^{1} \leqslant J_{2}$ при $\eta_{0} \leqslant \eta<1$. Возьмем $C_{5} \geqslant C_{8}$. Тогда при $\eta_{0} \leqslant \eta<1$ получим неравенство

$$
z^{1} \leqslant J_{1}^{1}
$$

Для разности $z^{k}-J_{1}=S^{k}$ имеем

$$
\begin{aligned}
P_{k}\left(J_{1}\right)- & P_{k}(z)=\nu\left(w^{k}\right)^{2} S_{\eta \eta}^{k}+G^{k}\left(w^{k}\right)^{4} S_{\eta \eta}^{k}-\eta \frac{k h V^{k}}{h} S^{k}+E^{k} S_{\eta}^{k}+F^{k} S_{\eta}^{k}+ \\
& +3 H^{k}\left(w^{k}\right)^{2}\left(S_{\eta}^{k}\right)^{3}+(\eta-1) B^{2} S_{\eta}^{k}+2 \nu w^{k} J_{1 \eta}^{k} S^{k}+2 \nu w^{k} z^{k} S_{\eta}^{k}+4 G^{k}\left(w^{k}\right)^{3} J_{1 \eta}^{k} S^{k}+ \\
+ & 4 G^{k}\left(w^{k}\right)^{3} z^{k} S_{\eta}^{k}+E_{\eta}^{k} S^{k}+2 H^{k}\left(w^{k}\right)^{3} J_{1 \eta}^{k} S^{k}+B^{2} S^{k}+2 H^{k}\left(w^{k}\right)^{3} z^{k} S_{\eta}^{k}<-\eta \frac{k h V^{k}}{h} S^{k-1} .
\end{aligned}
$$

В неравенстве (3.30) коэффициент при $S^{k}$ равен

$$
2 \nu w^{k} J_{1 \eta}^{k}+4 G^{k}\left(w^{k}\right)^{3} J_{1 \eta}^{k}+F^{k}+E_{\eta}^{k}+2 H^{k}\left(w^{k}\right)^{3} J_{1 \eta}^{k}-\eta k V^{k}-B^{2} k V^{k} .
$$

Если $0 \leqslant k h \leqslant X$ и $X$ достаточно мало, то выражение (3.31) отрицательно при $k \geqslant 2$ для $0 \leqslant \eta \leqslant 1$ и при $k=1$ для $0 \leqslant \eta \leqslant \eta_{0}$ в силу выбора $\eta_{0}$. Так как $S^{1}(0)>0, S^{1}\left(\eta_{0}\right)>0$, как показано выше, и $S^{0}=0$ в силу леммы 3.2 , то из (3.30) согласно принципу максимума получаем $S^{1}>0$ при $0 \leqslant \eta \leqslant \eta_{0}$. Вместе с (3.29) это приводит к неравенству $S^{1} \leqslant 0$ при $0 \leqslant \eta \leqslant 1$.

Далее, в силу того, что сумма (3.31) при $k \leqslant 2$ отрицательна, $S^{1} \leqslant 0$ при $0 \leqslant \eta<1$, из (3.30), (3.27) и (3.28) по принципу максимума выводим неравенство $S^{k} \leqslant 0$ при $0 \leqslant \eta<1, k \leqslant 2$. Поэтому

$$
z^{k} \leqslant J_{1}=Y_{\eta} e^{-C_{5} k h}
$$

Для оценки функции $z^{k}$ снизу рассмотрим функцию $J_{3}=Y_{\eta} e^{C_{4} k h}$ и вычислим $P_{k}\left(J_{3}\right)$. Так же, как при оценке $z^{k}$ сверху, покажем, что $P_{k}\left(J_{3}>0\right)$, если $C_{6}$ достаточно велико и $X$ достаточно мало. Выбрав $C_{4}>C_{2}$, получим

$$
\left.\left(J_{3}-z^{k}\right)\right|_{\eta=\bar{\eta}_{m}} \leqslant 0,\left.\quad\left(J_{3}-z^{k}\right)\right|_{\eta=0}<0
$$


при достаточно большом $C_{4}$ и $0 \leqslant k h \leqslant x$. Рассуждая далее так же, как при доказательстве оценки $z^{k}$ сверху, получим, что

$$
z^{k} \geqslant Y_{\eta} e^{C_{4} k h}
$$

Из полученных оценок функций $w^{k}, r^{k}, z^{k}$ и уравнения (3.3) следует ограниченность произведений $\left(w^{k}\right)^{2} w_{\eta \eta}^{k}$ и $\left(w^{k}\right)^{4} w_{\eta \eta}^{k}$. Установим второе из неравенств (3.22). Из (3.3) находим

$$
\begin{gathered}
\nu w^{k} w_{\eta \eta}^{k}\left(1+3 d(k h)^{2}\left(V^{k}\right)^{2}\left(w^{k}\right)^{3} w_{\eta \eta}^{k}\right)=\eta k h V^{k} \frac{r^{k}}{w^{k}}-\left(\eta^{2}-1\right)\left(V^{k}+k h V_{\xi}^{k}\right) \frac{w_{\eta}^{k}}{w^{k}}- \\
-\eta\left(V^{k}+k h V_{\xi}^{k}\right)-(\eta-1) B^{2} \frac{w_{\eta}^{k}}{w^{k}}+B^{2} k h V^{k}-H^{k}\left(w^{k}\right)^{2}\left(w_{\eta}^{k}\right)^{2} \leqslant \\
\leqslant \eta k h V^{k} \frac{r^{k}}{w^{k}}-\left(\eta^{2}-1\right) \frac{a Y_{\eta}}{Y}+\eta a-(\eta-1) B^{2} \frac{Y_{\eta}}{Y}+ \\
+\left(\eta^{2}-1\right)\left[\frac{a Y_{\eta}}{Y}-\left(V^{k}+k h V_{\xi}^{k}\right) \frac{w_{\eta}^{k}}{w^{k}}\right]-\left[\eta a-\eta\left(V^{k}+k h V_{\xi}^{k}\right)\right]-H^{k}\left(w^{k}\right)^{2}\left(w_{\eta}^{k}\right)^{2}< \\
<-\nu M_{6} M_{17}=-\nu C_{7},
\end{gathered}
$$

если $0 \leqslant k h \leqslant X$ и $X$ достаточно мало. Этим доказательство леммы 3.4 завершено.

Доказательство теоремы 3.1. Продолжим решения $w^{k}(\eta)$ задачи $(3.3),(3.4)$ линейно по $\xi$ при $(k-1) h \leqslant \xi \leqslant k h, k=1,2, \ldots, X / h$. В силу лемм $3.3,3.4$ полученное семейство функций $w_{h}(\xi, \eta)$ равномерно ограничено и равностепенно непрерывно в $\Omega$. По теореме Арцела получаем, что существует последовательность функций $w_{h_{m}}(\xi, \eta)$, равномерно сходящаяся в $\Omega$ к некоторой функции $w(\xi, \eta)$ при $h_{m} \rightarrow 0$. Точно так же, как в $[5, \S 3.1]$, доказывается, что $w$ имеет обобщенные производные, входящие в уравнение (2.4), и удовлетворяет этому уравнению почти всюду, а также неравенствам (3.1), (3.2).

Чтобы доказать, что производные функции $w$, входящие в уравнение, удовлетворяют условию Гельдера в любой внутренней подобласти области $\Omega$, рассмотрим в прямоугольнике $\Delta=\left\{\xi_{1} \leqslant\right.$ $\left.\xi \leqslant \xi_{2}, \eta_{1} \leqslant \eta \leqslant \eta_{2}\right\}$ уравнение

$$
\left.\nu\left(1+3 d \xi^{2} V\right)^{2} w^{2}\right) w^{2} S_{\eta \eta}-\eta \xi V_{\xi}^{S}+E S_{\eta}+F S+6 \nu d \xi^{2} V^{2} w^{3} S_{\eta}^{2}+(\eta-1) B^{2} S_{\eta}-B^{2} \xi V S=0
$$

с условиями

$$
\left.S\right|_{\xi=\xi_{1}}=\left.w\right|_{\xi=\xi_{1}},\left.\quad S\right|_{\eta=\eta_{1}}=\left.w\right|_{\eta=\eta_{1}},\left.\quad S\right|_{\eta=\eta_{2}}=\left.w\right|_{\eta=\eta_{2}}
$$

где $0<\xi_{1}<\xi_{2}<X, 0<\eta_{1}<\eta_{2}<1$. Поскольку $w$ удовлетворяет условию Липшица, то задача $(3.32),(3.33)$ в области $\Delta$ имеет такое решение $S$, что $S_{\eta}$ ограничено в $\Delta, S_{\eta}, S_{\xi}, S_{\eta \eta}$ удовлетворяют условию Гельдера в любой внутренней подобласти $\Delta$. Покажем, что $S=w$. Функция $W=S-w$ удовлетворяет уравнению

$$
\begin{aligned}
\nu\left(1+3 d \xi^{2} V^{2} w^{2}\right) w^{2} W_{\eta \eta}-\eta \xi V W_{\xi} & +E W_{\eta}+F W+ \\
& +6 \nu d \xi^{2} V^{2} w^{3} W_{\eta}^{2}+(\eta-1) B^{2} W_{\eta}-B^{2} \xi V W=0
\end{aligned}
$$

и условиям

$$
\left.W\right|_{\xi=\xi_{1}}=\left.W\right|_{\eta=\eta_{1}}=\left.W\right|_{\eta=\eta_{2}}=0 .
$$

Умножим уравнение (3.34) на $W e^{-\alpha \xi}, \alpha=$ const $>0$, и проинтегрируем по $\Delta$. Преобразуя некоторые члены интегрированием по частям, получим равенство

$$
\begin{aligned}
\int_{\Delta}\left[-\nu w^{2} W_{\eta}^{2}+\nu\left(w_{\eta}^{2}\right.\right. & \left.+w w_{\eta \eta}\right) W^{2}-G w^{4} W_{\eta}^{2}+2 G\left(3 w^{2} w_{\eta}^{2}+w^{3} w_{\eta \eta}\right) W^{2}-\eta B^{2} W^{2}- \\
-\frac{1}{2} B^{2} \xi V W & +\frac{1}{2} B^{2}(\xi V)_{\xi} W^{2}+\eta(\xi V)_{\xi} \frac{1}{2} W^{2}-\eta \xi V \frac{1}{2} W^{2} \alpha-\frac{1}{2} E_{\eta} W^{2}+F W^{2}- \\
& \left.-\frac{1}{2} H w^{3} W_{\eta \eta} W^{2}-\frac{3}{2} H w^{2} w_{\eta} W_{\eta} W^{2}\right] e^{-\alpha \xi} d \xi d \eta-\int_{\xi=\xi_{2}} \eta \xi V \frac{1}{2} W^{2} e^{-\alpha \xi} d \eta=0,
\end{aligned}
$$


а из него неравенство

$$
\begin{aligned}
& \int_{\Delta}\left[\nu\left(w_{\eta}^{2}+w w_{\eta \eta}\right)+2 G\left(3 w^{2} w_{\eta}^{2}+w^{3} w_{\eta \eta}\right)+\right. \\
& \left.\quad+\frac{1}{2} \eta(\xi V)_{\xi}-\frac{1}{2} \eta \xi V \alpha-\frac{1}{2} E_{\eta}+F-\eta B^{2}\right] W^{2} e^{-\alpha \xi} d \xi d \eta \geqslant 0 .
\end{aligned}
$$

При достаточно большом $\alpha$ выражение в квадратных скобках может быть сделано отрицательным, и тогда из (3.36) следует, что $W \equiv 0$ и $S=w$.

Обращая преобразование переменных (2.3), что возможно в силу свойств решения задачи (2.4), (2.5), получаем основной результат о существовании и единственности классического решения задачи (2.1), (2.2) в смысле данного нами определения.

Теорема 3.2. Предположим, что

$$
U(x)=x\left(a+x a_{1}(x)\right), \quad v_{0}(x)=b+x b_{1}(x),
$$

где $a=\mathrm{const}>0, b=\mathrm{const;} U(x)>0$ при $x>0 ; a_{1}(x), a_{1 x}(x), a_{1 x x}(x), b_{1}(x), b_{1 x}(x)$ ограниченз. Тогда задача (2.1), (2.2) в области $D$ при $X$, зависящем от $U$ u $v_{0}$, имеет решение $u$, $v$, которое обладает следующими свойствами:

(i) $u_{y}>0$ при $y \geqslant 0, x>0 ; u / U, u_{y} / U$ ограничены и непрерывны в $\bar{D}$;

(ii) $u>0$ nри $y>0, x>0 ; u(x, y) \rightarrow U(x)$ nрu $y \rightarrow \infty, u(x, 0)=u(0, y)=0$;

(iii) $u_{y} / U>0$ npu $y \geqslant 0 ; u_{y} / U \rightarrow 0$ npu $y \rightarrow \infty$;

(iv) $u_{x}, u_{y}, u_{y y}$ ограниченъ и непрерьвны в $\bar{D}$;

(v) $v$ непреръвна в $\bar{D}$ по у при $x>0$; $v$ непреръвна по $x$ и у внутри $D$;

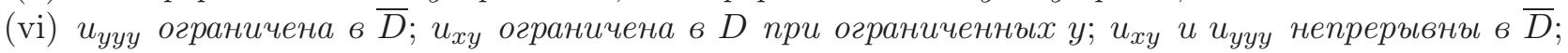
$u_{\text {yу }} / u_{y}$ непреръвна в $\bar{D}$ по $y$;

(vii) имеют место оценки

$$
\begin{gathered}
U(x) Y\left(\frac{u}{U}\right) e^{-C_{1} x} \leqslant u_{y} \leqslant U(x) Y\left(\frac{u}{U}\right) e^{C_{2} x}, \quad Y_{\eta}\left(\frac{u}{U}\right) e^{C_{4} x} \leqslant \frac{u_{y y}}{u_{y}} \leqslant Y_{\eta}\left(\frac{u}{U}\right) e^{-C_{5} x}, \\
\exp \left[-\frac{M_{1}^{2}}{4} y^{2} e^{2 C_{2} x}\right] \leqslant 1-\frac{u}{U} \leqslant \exp \left[-\frac{M_{1}^{2}}{4} y^{2} e^{-2 C_{1} x}\right],
\end{gathered}
$$

где $Y(\eta)$ - решение задачи (3.13), (3.14).

Доказательство теоремъ 3.2. Доказательство проводится на основе теоремы 3.1. Для начала покажем, что если $w(\xi, \eta)$ обладает свойствами, указанными в теореме 3.1, то можно с помощью замены (2.3) перейти от решения задачи (2.4), (2.5) к решению задачи $(2.1),(2.2)$, существование которого доказано в теореме 4.1. Согласно (2.3) имеем

$$
w(\xi, \eta)=w\left(x, \frac{u}{U}\right)=\frac{u_{y}}{U}, \quad x=\xi, \quad y=\int_{0}^{u(x, y) / U(x)} \frac{d s}{w(x, s)} .
$$

Отсюда в силу непрерывности функции $w(\xi, \eta)$ в $\bar{\Omega}$ и неравенства $w>0$ при $0 \leqslant \eta<1$ получаем, что $u(x, y) / U(x)$ непрерывна и ограничена в $\bar{D}$,

$$
u(0, y)=0, \quad u(x, 0)=0, \quad u(x, y) \rightarrow U(x) \quad \text { при } \quad y \rightarrow \infty,
$$

$u_{y}$ непрерывна и ограничена в $\bar{D}, u_{y}>0$ при $y \geqslant 0, x \geqslant 0$. Из (3.37) находим

$$
\begin{gathered}
u_{y}=w U, \quad \frac{u_{y y}}{u_{y}}=w_{\eta}, \quad u_{y y}=U w_{\eta} \eta_{y}=w_{\eta} u_{y}, \quad u_{y y y}=w_{\eta \eta} \frac{u_{y}^{2}}{U}+w_{\eta} u_{y y}, \\
u_{x y}=w U_{\xi}+U w_{\xi}+u_{\xi} w_{\eta}-u w_{\eta} \frac{U_{\xi}}{U}, \quad u_{x}=u \frac{U_{x}}{U}+w U \int_{0}^{u(x)} \int^{(x)} \frac{w_{\xi}(x, s)}{w^{2}(x, s)} d s .
\end{gathered}
$$


Из свойств функции $w$ и ее производных ввиду равенств (3.38) следует, что обобщенные производные $u_{x}, u_{y y}, u_{y y y}$ ограничены в $D, u_{x y}$ ограничена при конечных $y$. Неравенства для $u$, утверждаемые теоремой 4.1, следуют из оценок функций $w, w_{\xi}, w_{\eta}$ и $w w_{\eta \eta}$. Непрерывность $u_{x}$ и $u_{y y}$ по $y$ следует из (3.38). Функцию $v(x, y)$ определим равенством

$$
v=\frac{1}{u_{y}}\left(-u u_{x}+\nu u_{y y}\left(1+3 d u_{y}^{2}\right)+U U_{x}+B^{2} U-B^{2} u\right) .
$$

Покажем, что $u$ и $v$, определенные формулами (3.37), (3.39), удовлетворяют системе (2.1) и условиям (2.2). Функция $v$ имеет производную по $y$ в $D$. Дифференцируя (3.39) по $y$, получим

$$
v_{y} u_{y}+v u_{y y}+u_{y} u_{x}+u u_{x y}-\nu u_{y y y}-6 \nu d u_{y} u_{y y}^{2}-3 \nu d u_{y}^{2} u_{y y y}-B^{2} u_{y}=0
$$

или

$$
\begin{aligned}
v_{y} u_{y}+\frac{u_{y y}}{u_{y}}\left(-u u_{x}+\nu u_{y y}\left(1+3 d u_{y}^{2}\right)+U U_{x}\right) & +u_{y} u_{x}+u u_{x y}-\nu u_{y y y}- \\
& -6 \nu d u_{y} u_{y y}^{2}-3 \nu d u_{y}^{2} u_{y y y}-B^{2} u_{y}=0 .
\end{aligned}
$$

Функция $w(\xi, \eta)=u_{y} / U$ удовлетворяет уравнению (2.4). Заменяя в уравнении (2.4) производные $w$ через производные от $u$, находим, что

$$
\begin{aligned}
& \frac{1}{U}\left\{\nu\left(1+3 d u_{y}^{2}\right) \frac{u_{y y y} u_{y}-u_{y y}^{2}}{u_{y}}-\frac{\left(u_{x y} u_{y}-u_{x} u_{y y}^{2}\right) u}{u_{y}}-\frac{u U_{x}\left(u u_{y y}-u_{y}^{2}\right)}{U u_{y}}+\right. \\
& \left.\quad+\frac{\left(u^{2}-U^{2}\right) U_{x}}{U} \frac{u_{y y}}{u_{y}}+u u_{y}-\frac{u u_{y} U_{x}}{U}+6 \nu d u_{y y}^{2} u_{y}+(u-U) \frac{u_{y y}}{u_{y}} B^{2}-B^{2} u_{y}\right\}=0 .
\end{aligned}
$$

Умножив (3.41) на $U$ и складывая равенства (3.40) и (3.41), получим

$$
u_{x}+u_{y}=0 .
$$

Уравнения (3.39), (3.42) представляют систему (2.1). Покажем, что $v(x, y)$ удовлетворяет условию $v(x, 0)=v_{0}(x)$. Из $(2.5)$ следует, что

$$
v_{0}=\left.\left[\frac{\nu w w_{\eta}\left(1+3 d U^{2} w^{2}\right)+U_{x}+B^{2}}{w}\right]\right|_{\eta=0} .
$$

Используя (3.39), получаем

$$
\begin{aligned}
v(x, 0)=\left.\left[\frac{\nu u_{y y}\left(1+3 d u_{y}^{2}\right)+U U_{x}+B^{2} U+B^{2} u}{u_{y}}\right]\right|_{y=0}= & \\
& {\left.\left[\frac{\nu w w_{\eta}\left(1+3 d U^{2} w^{2}\right)+U_{x}+B^{2}}{w}\right]\right|_{\eta=0}=v_{0}(x) . }
\end{aligned}
$$

Здесь мы использовали непрерывность функций $u, u_{x}, u_{y}, u_{y y}$ по $y$ при $y=0$, а также непрерывность $w$ и $w w_{\eta}$ по $\eta$. Как следует из (3.41), функция $v$, определенная равенством (3.39), непрерывна в $\bar{D}$ по $y$ и ограничена при ограниченных $y$; $v_{y}$ ограничена в $D$, так как $v_{y}=-u_{x}$, а $u / U$ и $u$ ограничены.

Выведем асимптотическую формулу для отношения $u(x, y) / U(x)$ при $y \rightarrow \infty$. Используя оценки функций $w(\xi, \eta)$ из теоремы 3.1 и оценки $Y(\eta)$ из леммы 3.2 , получаем при $\eta_{0} \leqslant \eta \leqslant 1$ неравенства

$$
M_{1}(1-\eta)\left(\sigma-K_{4}\right) e^{-C_{1} x} \leqslant w(\xi, \eta) \leqslant M_{1}(1-\eta) \sigma e^{C_{2} x} .
$$

Это приводит к неравенствам

$$
M_{1}\left(1-\frac{u}{U}\right)\left(\sigma\left(\frac{u}{U}\right)-K_{4}\right) e^{-C_{1} x} \leqslant \frac{u_{y}}{U} \leqslant M_{1}\left(1-\frac{u}{U}\right) \sigma\left(\frac{u}{U}\right) e^{C_{2} x} .
$$

Из того факта, что

$$
\sigma_{y}=\frac{u_{y} / U}{2(1-u / U) \sigma}
$$


получаем

$$
\frac{2 \sigma_{y}}{M_{1} e^{C_{2} x}} \leqslant 1 \leqslant \frac{2 \sigma \sigma_{y}}{M_{1}\left(\sigma-K_{4}\right) e^{-C_{1} x}} .
$$

Интегрируя последние неравенства по $y$ от $y_{0}$, соответствующего $\eta_{0}$, до произвольного $y \in\left(y_{0}, \infty\right)$, получаем

$$
\frac{2\left(\sigma-\sigma_{0}\right)}{M_{1} e^{C_{2} x}} \leqslant y-y_{0} \leqslant \frac{2\left(\sigma-\sigma_{0}\right)}{M_{1} e^{-C_{1} x}}+\frac{2 K_{4}}{M_{1} e^{-C_{1} x}}\left[\ln \left(\sigma-K_{4}\right)-\ln \left(\sigma_{0}-K_{4}\right)\right]
$$

где $\sigma=\sqrt{-\ln \mu(1-u / U)}, \sigma_{0}=\left.\sigma\right|_{y=y_{0}}$.

Из этих неравенств находим

$$
\frac{\left(y-y_{0}\right) M_{1} e^{-C_{1} x}}{2}+K_{4} \ln \left(1+\frac{\left(y-y_{0}\right) M_{1} e^{C_{2} x}}{2\left(\sigma-K_{4}\right)}\right)+\sigma_{0} \leqslant \sigma \leqslant \frac{\left(y-y_{0}\right) M_{1} e^{C_{2} x}}{2}+\sigma_{0}
$$

и, следовательно,

$$
\exp \left[-\frac{U(0)}{2 \nu} y^{2} e^{2 C_{2} x}+O(y)\right] \leqslant 1-\frac{u}{U} \leqslant \exp \left[-\frac{U(0)}{2 \nu} y^{2} e^{-2 C_{1} x}+O(y \ln y)\right] \quad \text { при } \quad y \rightarrow \infty,
$$

где $M_{1}^{2}=2 a / \nu, a=U_{x}(0)$. Данные неравенства доказывают справедливость формулы

$$
\exp \left[-\frac{M_{1}^{2}}{4} y^{2} e^{2 C_{2} x}\right] \leqslant 1-\frac{u}{U} \leqslant \exp \left[-\frac{M_{1}^{2}}{4} y^{2} e^{-2 C_{1} x}\right] .
$$

\section{4. Теорема о единственности решения задачи.}

Теорема 4.1. Задача (2.4), (2.5) в области $\Omega$ может иметь лишь одно неотрииательное решение $w$, обладающее следуюшими свойствами: $w$ непрерывна в $\Omega ; w_{\eta}, w_{\eta \eta}$, $w_{\xi}$ непрерьвнь во внутренних точках $\Omega ; w>0$ при $\eta=0 ; w_{\eta}$ непрерьвна по $\eta$ при $\eta=0$.

Доказательство теоремъ 3.2. Предположим, что задача имеет два таких решения $w_{1}$ и $w_{2}$. Их разность $\bar{w}=w_{1}-w_{2}$ удовлетворяет уравнению

$$
\begin{aligned}
\nu w_{1}^{2} \bar{w}_{\eta \eta}+\nu\left(w_{1}+w_{2}\right) w_{2 \eta \eta} \bar{w}+3 \nu d \xi^{2} V^{2} w_{1}^{4} \bar{w}_{\eta \eta}+3 \nu d \xi^{2} V^{2}\left(w_{1}^{3}+w_{1} w_{2}^{2}+w_{1}^{2} w_{2}+w_{2}^{3}\right) w_{2 \eta \eta} \bar{w}- \\
-\eta \xi V \bar{w}_{\xi}+\left(\eta^{2}-1\right)\left(V+\xi V_{\xi}\right) \bar{w}_{\eta}-\eta\left(V+\xi V_{\xi}\right) \bar{w}+6 \nu d \xi^{2} V^{2}\left(w_{1 \eta}+w_{2 \eta}\right) w_{2}^{3} \bar{w}_{2 \eta}+ \\
+6 \nu d \xi^{2} V^{2}\left(w_{1}^{2}+w_{1} w_{2}+w_{2}^{2}\right) w_{1 \eta}^{2} \bar{w}+(\eta-1) B^{2} \bar{w}_{\eta}-B^{2} \xi V \bar{w}=0
\end{aligned}
$$

с граничными условиями

$$
\left.\bar{w}\right|_{\eta=1}=0,\left.\quad\left(\nu \bar{w}_{\eta}+3 \nu d \xi^{2} V^{2} w_{1}^{2} \bar{w}_{2 \eta}+3 \nu d \xi^{2} V^{2}\left(w_{1}+w_{2}\right) w_{2 \eta} \bar{w}-\frac{\left(V+\xi V_{\xi}\right) \bar{w}}{w_{1} w_{2}}-\frac{B^{2} \bar{w}}{w^{1} w^{2}}\right)\right|_{\eta=0}=0 .
$$

Для $W=\bar{w} e^{-\alpha \xi}, \alpha=$ const $>0$, имеем следующее уравнение в области $\Omega$ :

$$
\begin{gathered}
\nu w_{1}^{2} W_{\eta \eta}+\nu\left(w_{1}+w_{2}\right) w_{2 \eta \eta} W+3 \nu d \xi^{2} V^{2} w_{1}^{4} W_{\eta \eta}+3 \nu d \xi^{2} V^{2}\left(w_{1}^{3}+w_{1} w_{2}^{2}+w_{1}^{2} w_{2}+w_{2}^{3}\right) w_{2 \eta \eta} W- \\
-\eta \xi V W_{\xi}-\alpha \eta \xi V W_{\xi}+\left(\eta^{2}-1\right)\left(V+\xi V_{\xi}\right) W_{\eta}-\eta\left(V+\xi V_{\xi}\right) W+ \\
+6 \nu d \xi^{2} V^{2}\left(w_{1}^{2}+w_{1} w_{2}+w_{2}^{2}\right) w_{1 \eta}^{2} W+6 \nu d \xi^{2} V^{2}\left(w_{1 \eta}+w_{2 \eta}\right) w_{2}^{3} W_{\eta}^{2}+(\eta-1) B^{2} W_{\eta}=0
\end{gathered}
$$

а также граничные условия

$$
\left.W\right|_{\eta=1}=0,\left.\quad\left(\nu W_{\eta}+3 \nu d \xi^{2} V^{2} w_{1}^{2} W_{\eta}+3 \nu d \xi^{2} V^{2}\left(w_{1}+w_{2}\right) w_{2 \eta} W-\frac{\left(V+\xi V_{\xi}+B^{2}\right) W}{w_{1} w_{2}}\right)\right|_{\eta=0}=0 .
$$

При достаточно большом $\alpha$ коэффициент при $W$ в $(4.1)$ отрицателен, $\left(V+\xi V_{\xi}\right)>0$ по условию. По принципу максимума из (4.1), (4.2) следует, что $W$ не может иметь ни положительного максимума, ни отрицательного минимума при $0 \leqslant \eta<1$. Следовательно, $W \equiv 0$ и $\bar{w} \equiv 0$.

Теорема 4.2. Пусть $u, v$-решение задачи (2.1), (2.2), удовлетворяющее следуюшим условиям: 


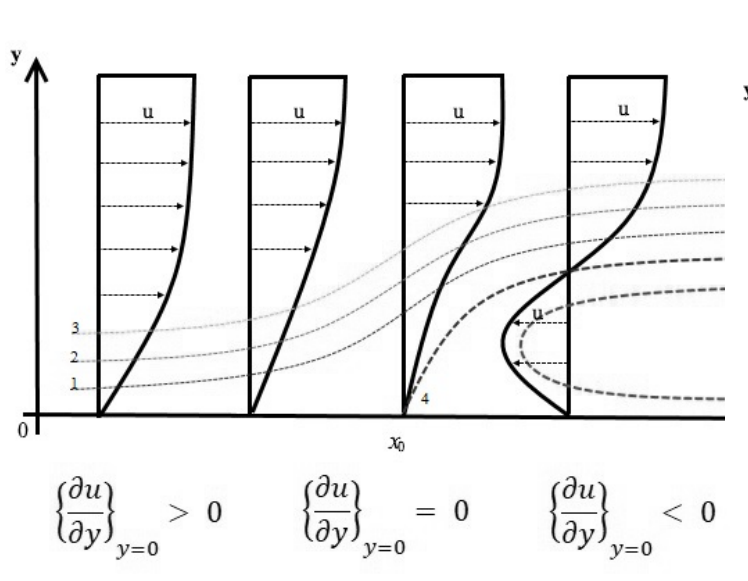

Рис. 1

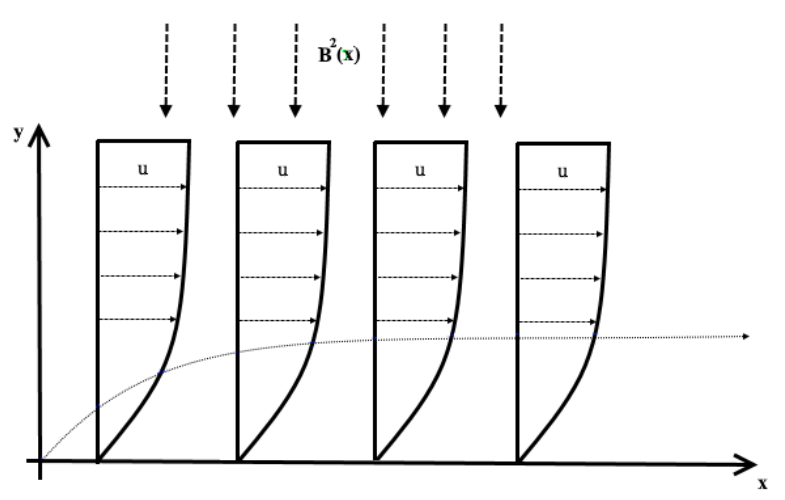

Рис. 2

(1) производные $u_{x}, u_{y}, v_{y}, u_{y y}, u_{y y y}, u_{x y}$ непрерывны в $D$;

(2) функции и/U и и $u_{y} / U$ непрерывны в $\bar{D}$;

(3) $u_{y}>0$ nрu $y \geqslant 0, x>0 ; u_{y} / U>0$ npu $y=0 ; u_{y} / U \rightarrow 0$ npu $y \rightarrow \infty$;

(4) $u_{y y} / u_{y}, u_{x}$ непрерьвны по у при $y=0$;

(5) $\frac{u_{y y y} u_{y}-u_{y}^{2}}{u_{y}^{2}} \leqslant 0$.

Тогда и, v-единственное решение задачи (2.1), (2.2) с указанными свойствами.

Доказательство теоремъ 4.2. Если $u, v$-решение задачи (2.1), (2.2), обладающее этими свойствами, то с помощью замены независимых переменных (2.3) и введения новой неизвестной функции $w=u_{y} / U$ приходим к решению $w$ задачи $(2.4),(2.5)$, обладающему свойствами из теоремы 3.1 , аналогично доказательству теоремы 4.1. Как показано выше, такое решение $w$ единственно.

5. Влияние магнитного поля на отрыв пограничного слоя. Одним из способов активного воздействия на характеристики пограничного слоя является искусственное воздействие поперечным магнитным полем на жидкости. Поперечное магнитное поле используется для управления точкой отрыва пограничного слоя, так как преждевременный его отрыв уменьшает подъемную силу и увеличивает лобовое сопротивление обтекаемого тела, например, профиля крыла или иной несущей поверхности.

Определение 5.1. Точкой отрыва пограничного слоя называется такая точка $x_{0}$, что $u_{y}\left(x_{0}, 0\right)=0$ и $u_{y}(x, 0)>0$ при $0<x<x_{0}$. Тогда $x_{0}$-верхняя грань таких $X>0$, что в области $D$ задача $(2.1),(2.2)$ имеет такое решение $u(x, y), v(x, y)$, что $u_{y}(x, 0)>0$.

В точке $x_{0}$ при $y=0$ производная скорости по $y$ равна нулю, откуда появляется перегиб, и устойчивость течения нарушается. С последующим поступательным движением пограничного слоя появляется область, где производная по у становится меньше нуля, т.е. возникает обратное течение жидкости и образуются вихри. При этом нарушается ламинарный характер течения и его стационарность. Этого можно избежать, подействовав магнитным полем, направленным перпендикулярно потоку жидкости. Под этим влиянием точка отрыва пограничного слоя от твердой обтекаемой поверхности сдвинется вправо, тем самым выравнивая течение жидкости (см. рис. 2). Таким образом, сделан вывод о том, что при достаточно сильном магнитном поле возможно безотрывное течение независимо от других параметров движения вязкой сплошной среды. 


\section{СПИСОК ЛИТЕРАТУРЫ}

1. Булатова Р. Р., Самохин В. Н., Чечкин Г. А. Уравнения магнитогидродинамического пограничного слоя для модифицированной несжимаемой вязкой среды. Отрыв пограничного слоя// Пробл. мат. анал. - 2018. - 92. - С. 83-100.

2. Булатова P. Р. Влияние магнитного поля на положение точки отрыва пограничного слоя электропроводной жидкости // Изв. вузов. Пробл. полиграфии и издат. дела. - 2018. - № 1. - С. 14-22.

3. Самохин В. Н., Фадеева Г. М., Чечкин Г. А. Уравнения пограничного слоя для модифицированной системы Навье-Стокса// Тр. семин. им. И. Г. Петровского. - 2011. - 28. - С. 329-361.

4. Самохин В. Н., Чечкин Г. А. Уравнения пограничного слоя обобщенно ньютоновской среды в окрестности критической точки// Тр. семин. им. И. Г. Петровского. - 2016. - 31. - С. 158-176.

5. Олейник O. А., Самохин B. Н. Математические методы в теории пограничного слоя. - М.: Наука, 1997.

6. Bulatova R. R., Chechkin G. A., Chechkina T. P., Samokhin V. N. On the influence of a magnetic field on the separation of the boundary layer of a non-Newtonian MHD medium// C. R. Méc. — 2018. — 346, № 9. - P. 807-814.

Булатова Регина Рашидовна

Московский государственный университет им. М. В. Ломоносова

E-mail: regina.bulatova@mech.math.msu.su 